\title{
Enhancement of jet fuel range alkanes from co-feeding of lignocellulosic biomass with plastics via tandem catalytic conversions
}

(1)

Xuesong Zhang, Hanwu Lei*, Lei Zhu, Moriko Qian, Xiaolu Zhu, Joan Wu, and Shulin Chen. Bioproducts, Sciences and Engineering Laboratory, Department of Biological Systems Engineering, Washington State University, Richland, WA 99354-1671, USA. *Corresponding author, Phone: 509-372-7628, Fax: 509-372-7690, E-mail: hlei@wsu.edu

ABSTRACT: Enhanced carbon yields of jet fuel range alkanes were manufactured from cofeeding of lignocellulosic biomass with plastics. The consecutive processes proceeded via the cofeed catalytic microwave-induced pyrolysis and hydrogenation process. In the co-feed catalytic microwave pyrolysis by using ZSM-5 as the catalyst, parent ZSM-5 fabricated by hydrothermal and calcined treatments contributed to the increase of surface area as well as the formation of more mesopores. Liquid organics with enhanced carbon yield (40.54\%) were more principally lumped in the jet fuel range from the co-feed catalytic microwave pyrolysis performed at the catalytic temperature of $375^{\circ} \mathrm{C}$ with the plastics to biomass ratio of 0.75 . To manufacture homemade Raney Ni catalyst, the BET surface area, pore surface area, and pore volume of the homemade Raney Ni catalyst were considerably improved when the Ni-Al alloy was dissolved by the $\mathrm{NaOH}$ solution. In the hydrogenation process, we observed the three species of raw organic derived from the co-feed catalytic microwave pyrolysis were almost completely converted into saturated hydrocarbons under a low-severity condition. The improved carbon yield (38.51\%) of hydrogenated organics regarding co-reactants of biomass and plastics predominantly match jet fuels. In the hydrogenated organics, over $90 \%$ selectivity toward alkanes with the carbon number in the jet fuel range was attained. In this respect, these hydrogenated organics with high amounts of renewable cycloalkanes can be potentially served as high-density jet fuels or additives for 
blending with civilian jet fuels.

3 KEYWORDS: Bio-jet fuels; well-promoted ZSM-5; home-made Raney Ni; co-feed catalytic

4 microwave pyrolysis; hydrogenation; cycloalkanes

\section{$5 \quad 1$ Introduction}

6 The limitation of fossil resources accompanied with huge environmental issues accelerate the

7 exploitation of renewable resources to substitute petroleum hydrocarbon fuels, with a particular 8 concentration on the development of new generation biofuels [1-3]. Lignocellulosic biomass has

9 attracted essential attention as a carbon-neutral resource for promising potentials to produce 10 transportation biofuels and versatile chemicals [4, 5]. Catalytic fast pyrolysis (CFP) of 11 lignocellulose to valuable biofuels and chemicals is recognized as the most prevailing and 12 promising route in a single process [6-11]. Dozens of lignocellulose and biomass-derived 13 feedstock have been subjected to catalytic pyrolysis for the production of advanced biofuels with 14 more favorable properties [6-8, 12, 13]. Zeolite-based catalysts (e.g., ZSM-5) have been 15 recognized as the highest-efficiency catalysts to produce considerable petrochemicals (aromatics 16 and olefins) $[9,14,15]$.

18 However, even in the presence of highly efficient catalyst, catalytic pyrolysis of lignocellulose 19 can solely manufacture low carbon yield $(10-30 \%)$ of aromatic hydrocarbons; large amounts of 20 solid residues, including both biochar and coke (carbon yields usually above 30\%), are achieved 21 in the process $[10,16-19]$. As aromatics are considered as the high energy-density hydrocarbons 
production [11], such low carbon yield of aromatic hydrocarbons from catalytic pyrolysis is not cost-effective to scale up the process in a biorefinery. Further, the process is commonly plagued by the high production of coke deposited on the catalyst because the coke could rapidly deactivate the catalyst and reduce its lifetime, resulting in the catalytic process to be impractical[20]. Therefore, these are the huge challenges in the face of commercializing the catalytic pyrolysis for the production of renewable petrochemicals.

(1)

It is discerned that the petrochemicals with the low carbon yield of aromatics and high coke formation are mainly associated with the oxygen-enriched intrinsic nature and hydrogen deficiency of lignocellulose $[16,18,21]$. In addition, the hydrogen to carbon effective $\left(\mathrm{H} / \mathrm{C}_{\text {eff }}\right)$ ratio plays a significant role in coke formation and converting efficiency of biomass into advanced biofuels $[8,22,23]$. Thus the hydrogen-deficient $\left(\mathrm{H} / \mathrm{C}_{\text {eff }}\right.$ usually less than 0.3$)$ biomass produces low carbon yields of petrochemicals and large formation of coke when the lignocellulose were transformed over zeolite catalysts $[9,15]$. In order to improve the carbon efficiency of aromatics and minimize the coke formation, it is reasonable that the incorporation of high $\mathrm{H} / \mathrm{C}_{\text {eff }}$ ratio co-reactants with biomass in the catalytic pyrolysis could help mitigate these issues. It is observed that co-feeding of lignocellulose with hydrogen-rich feedstock in the catalytic pyrolysis can modify the reaction mechanism of oxygen removal by substituting decarbonylation and decarboxylation with dehydration [10, 22, 24, 25]. Waste plastics represent a cheaper and abundant hydrogen sources, which can be used to improve carbon efficiency of aromatics and lower the coke formation in the catalytic co-pyrolysis $[9,15,18,21,26]$. Tremendous quantities of waste plastics are generated each year worldwide. Among the waste plastics, polyethylene formed by the polymerization of olefins with a $\mathrm{H} / \mathrm{C}_{\text {eff }}$ ratio of 2 accounts 
1 for up to $40 \%$ of gross waste plastics [27]. Hence co-feeding of lignocellulose with waste plastics

2 in catalytic pyrolysis is remarkably beneficial for the environment and energy recapture.

4 Although the co-feeding of biomass with plastics in catalytic pyrolysis has a significant synergy

5 for aromatic production and coke reduction, these aromatics are commonly mono-ring aromatic

6 hydrocarbons with low carbon numbers $\left(\mathrm{C}_{6}-\mathrm{C}_{8}\right)[21,23,25,26]$. Yet, these aromatics with low

7 carbon numbers cannot satisfy the requirement of jet fuels [19]; that is due to the fact that the

8 current jet fuels are principally comprised of linear-chain and branched-chain alkanes in the

9 range of $\mathrm{C}_{8}-\mathrm{C}_{16}[28,29]$. It is well known that cycloalkanes in jet fuels can be burned cleanly

10 with high heats of combustion; that is because cycloalkanes are compact molecules within robust

11 ring strain [30, 31]. To promote the qualities of current jet fuels, cycloalkanes with the carbon

12 numbers in the jet fuel range can be synthesized and added into civilian jet fuels [29, 32]. The

13 representative techniques on bio-jet fuel production include catalytic hydrodeoxygenation of

14 vegetable oils [33, 34] and Fischer-Tropsch synthesis of biomass-derived syngas [5, 11].

15 Nonetheless, aromatics and cycloalkanes cannot be generated by the two technologies. On the

16 other hand, the promising pathway for the production of cycloalkanes are the hydro-

17 cycloaddition of aromatic hydrocarbons [35-38]. To pursue the precursors of renewable

18 cycloalkanes for jet fuels, highly desirable aromatic hydrocarbon in the jet fuel range $\left(\mathrm{C}_{8}-\mathrm{C}_{16}\right)$

19 should be generated.

21 Due to conventional ZSM-5 zeolite with microporous structure, it contributes to mono-ring 22 aromatics with low carbon numbers. In our previous study, moderate treatments were used to 
fabricate ZSM-5 properties including its porosity and acidity, which is vital for improving

2 product selectivity toward aromatic hydrocarbons [39, 40]. It was shown that the mild

3 hydrothermal and calcined modifications favored the generation of mesopores and more catalytic

4 sites. Accordingly our well-modified catalyst is suitable for the production of aromatic

5 hydrocarbons in the $\mathrm{C}_{8}-\mathrm{C}_{16}$ range. Besides we have developed a combined process to

6 manufacture renewable cycloalkane from lignocellulosic biomass, albeit obtaining a low carbon

7 yield [35]. In addition, it is manifested that microwave-assisted pyrolysis technology is one of

8 the most promising techniques to enhance the degradation reactions because of rapid heat rate by

9 microwave irradiation [41]. In comparison with conventional pyrolysis, microwave-assisted

10 pyrolysis encloses the advantages of rapid heating, easy control, and low energy input [42, 43].

11 In the hydro-cycloaddition process, the catalytic performance of home-made Raney Ni catalyst

12 was superior to purchased Raney Ni catalysts, producing higher yields of cycloalkanes under the

13 same system [35]. During the process, the aromatics in the $n$-heptane medium were almost

14 completely converted into saturated hydrocarbons under a low-severity condition.

16 Initially, the production of $\mathrm{C}_{8}-\mathrm{C}_{16}$ aromatics was the target compounds from the co-feeding of 17 lignocellulosic biomass and plastics in the catalytic pyrolysis. To best of our knowledge, the co18 feed catalytic microwave-induced pyrolysis has not yet been explored. The main objective of this 19 research presented in this paper was the production of jet fuel range alkanes with enhanced 20 carbon yield. In this respect, there is no literature reported on the production of jet fuel range 21 alkanes from the co-feeding of lignocellulosic biomass with plastics. Furthermore, the reaction 22 mechanisms regarding the co-feeding of lignocellulosic biomass with plastics through catalytic 23 combined conversions have never been established. Therefore, this study can fill this research 
gap in the production of jet fuels from co-feeding of lignocellulosic biomass and plastics via tandem catalytic processes.

4 The innovations aspects of this study were the novel pathways from co-feeding of lignocellulosic

5 biomass with plastics by using modified catalysts, namely well-promoted ZSM-5 catalyst and

6 home-made Raney Ni catalyst with the purpose of enhancing the carbon yield of aromatics and

7 lowering the coke formation and improving the carbon selectivity of jet fuel range alkanes.

8 Herein, biomass and plastics were co-pyrolyzed using a tandem microwave-induced pyrolysis

9 system and a catalytic system, aiming to determine whether there is an apparent synergy between

10 co-feeding of biomass and plastics in the catalytic microwave-induced pyrolysis. For maximizing

11 the carbon efficiency of jet fuel range alkanes, the various species of bio-oils derived from co-

12 feed catalytic microwave-assisted pyrolysis was first extracted by the optimum solvent $(n-$

13 heptane). The organic mixture was subsequently hydrogenated into saturated hydrocarbons by

14 using home-made Raney Ni catalyst. The influence of organic species on the carbon yield and

15 product distribution of saturated hydrocarbon in the jet fuel range was also evaluated.

16

\section{Experimental sections}

\subsection{Materials}

19 Douglas fir sawdust pellets ( $7 \mathrm{~mm}$ in diameter and $15 \mathrm{~mm}$ in length) were leveraged as

20 lignocellulosic biomass, which were supplied by Bear Mountain Forest Products Inc., USA.

21 Low-density polyethylene pellets (LDPE) was purchased from Sigma-Aldrich Corporation (St.

22 Louis, MO, USA). Its density and melting point are $0.925 \mathrm{~g} / \mathrm{cm}^{3}$ and $116{ }^{\circ} \mathrm{C}$, respectively. The 
1 elemental compositions of Douglas fir sawdust pellets and LDPE are listed in Table 1. Parent

2 ZSM-5 $\left(\mathrm{SiO}_{2} / \mathrm{Al}_{2} \mathrm{O}_{3}\right.$ Mole Ratio: 50) was supplied by Zeolyst International, USA. Nickel-

3 Aluminum alloy powder in a non-activated form was purchased from Alfa Aesar (Ward Hill,

4 MA, USA).

5

\section{$6 \quad 2.2$ Catalyst preparation}

7 The parent ZSM-5 was fabricated by hydrothermal and calcined treatments. Parent ZSM-5

8 powder was added into deionized water (mass ratio $=1$ ) at $60{ }^{\circ} \mathrm{C}$ under the gentle stirring. The

9 slurry was kept at this condition for $2 \mathrm{~h}$. The slurry was subsequently dried at $105{ }^{\circ} \mathrm{C}$ until the

10 weight was constant. The sequential process was calcination: parent ZSM-5 tailored by

11 hydrothermal treatment was calcined at $550^{\circ} \mathrm{C}$ for $5 \mathrm{~h}$ in a muffle furnace. The obtained solid 12 was pelletized and sieved to $20-40$ mesh. For the regeneration of promoted ZSM-5, the spent 13 catalysts were burned at $550^{\circ} \mathrm{C}$ for $2 \mathrm{~h}$ in a muffle as well.

Home-made Raney Ni catalyst was fabricated by using a $20 \mathrm{wt} \% \mathrm{NaOH}$ aqueous solution to dissolve $\mathrm{Al}$ compostion in the $\mathrm{Ni}-\mathrm{Al}$ alloy powder. In this case, $10 \mathrm{~g}$ of $\mathrm{Ni}-\mathrm{Al}$ alloy powder was slowly added into $20 \mathrm{wt} \% \mathrm{NaOH}$ aqueous solution $(100 \mathrm{~mL})$ under gentle stirring. After addition,

18 the slurry was held on stirring at $80{ }^{\circ} \mathrm{C}$ for $1 \mathrm{~h}$. The excess of $\mathrm{NaOH}$ solution was consequently 19 washed by distilled water until neutral $\mathrm{pH}$ was reached. Since Raney Ni is notorious for its 20 pyrophoricity, it can ignite spontaneously when it is exposed in air. The obtained home-made 21 Raney Ni catalyst was therefore stored in water. Prior to the subsequent catalytic test, the Raney $22 \mathrm{Ni}$ catalyst was dried at $60{ }^{\circ} \mathrm{C}$ in the atmosphere of nitrogen to avoid contact with air. 
The schematic diagram of the microwave-assisted pyrolysis system integreated with the catalysis

4 system was presented in Fig. S1. Detailed experimental description was shown in our previous research $[44,45]$. Prior to the experiments, Douglas fir sawdust pellets were air dried at $105{ }^{\circ} \mathrm{C}$

6 for $24 \mathrm{~h}$ to remove the physically bound moisture. The co-feeding of Douglas fir sawdust pellets

7 with LDPE pellets were placed in a $500 \mathrm{~mL}$ quartz flask, which was put inside a Sineo MAS-II

8 batch microwave oven (Shanghai, China) by a constant microwave power setting (700 W). 0.05

$9 \mathrm{~g}$ of activated carbon powder was leveraged as microwave absorbers for the microwave-assisted pyrolysis. All runs for microwave-assisted pyrolysis in the microwave oven chamber were 11 implemented at $480{ }^{\circ} \mathrm{C}$ for $10 \mathrm{~min}$.

\subsection{Hydrogenation of liquid organics derived from co-feed catalytic microwave pyrolysis}

14 Due to the solvents influence in the hydro-cycloaddition of a model compound (naphthalene) in our previous study [35], the mixed bio-oils from co-feed catalytic microwave pyrolysis were extracted by the optimal solvent ( $n$-heptane). For the hydrogenation process of extracted organics,

17 a closed reaction system with a stirred stainless batch reactor of the 4592 micro stirred reactor 18 (with a $50 \mathrm{~mL}$ vessel) and a 4848 reactor controller from Parr Instrument Company (Moline, IL, 19 USA) was employed (Fig. S2). Herein, the mixture of organics and the $n$-heptane medium was 20 placed into the vessel with $20 \mathrm{wt} \%$ home-made Raney Ni catalyst (with respect to the reactants).

21 Then the reactor was sealed and vented for three times by hydrogen to expel the air in the vessel. 22 Hydrogen was subsequently adjusted to the set pressure (500 psi). The automatic controller was 


(6)

used to control the temperature and the revolution of stirrer $(350 \mathrm{rpm})$. The pressure inside the reactor was recorded and the reactions were conducted at $200{ }^{\circ} \mathrm{C}$ for $2 \mathrm{~h}$. After the experiment was quenched, stirring was stopped and the vessel was rapidly cooled to ambient temperature. Then, the gas was collected by a $1 \mathrm{~L}$ Tedlar gas bag and the reactor was depressurized. Consequently the liquid products were filtered to remove catalyst particles.

\subsection{Analytical techniques}

Elemental analysis $(\mathrm{C}, \mathrm{H}$ and $\mathrm{N})$ of reactants, liquid samples, char, and coke deposited on spent catalysts was measured by using a 2400 Series II CHN/O Elemental Analyzer (PerkinElmer, USA).

The textural properties of the catalysts were evaluated in light of $\mathrm{N}_{2}$ adsorption-desorption (Micromeritics TriStar II 3020 Automatic Physisorption Analyzer). Fresh catalysts (wellpromoted ZSM-5 catalyst and home-made Raney Ni catalyst) were degassed in vacuum at $300{ }^{\circ} \mathrm{C}$ for $1 \mathrm{~h}$. The Brunauer-Emmett-Teller equation was utilized to figure out the surface area by using adsorption data at $p / p o=0.05-0.25$. The pore volume was measured through the Barrett-Joyner-Halenda (BJH) method.

The acidity was determined by temperature-programmed desorption (TPD) of ammonia with a Micromeritics AutoChem II 2920 Chemisorption Analyzer equipped with a PFEIFFER mass spectrometer. The samples were initially saturated with $\mathrm{NH}_{3}$ at the room temperature in a flow of $10 \% \mathrm{NH}_{3}$ in nitrogen. After $\mathrm{NH}_{3}$ saturation, the weakly bound $\mathrm{NH}_{3}$ was desorbed prior to the 
9 The particle size and surface morphology of the samples were evaluated with a scanning electron

measurement at $120^{\circ} \mathrm{C}$ for $1 \mathrm{~h}$ at a He flow rate of $25 \mathrm{~mL} / \mathrm{min}$. The desorption curve was finally calculated at a heating ramp of $10{ }^{\circ} \mathrm{C} / \mathrm{min}$ from $120{ }^{\circ} \mathrm{C}$ to $550{ }^{\circ} \mathrm{C}$ with a He flow rate of 25 $\mathrm{ml} / \mathrm{min}$.

Powder X-ray diffraction (XRD) patterns were applied on a Rigaku Smartlab X-ray diffractometer equipped with a $\mathrm{Cu} \mathrm{K} \alpha \mathrm{X}$-ray source, which was operated at $40 \mathrm{kV}$ and $40 \mathrm{~mA}$. The scattering angle $2 \theta$ was changed from $10^{\circ}$ to $80^{\circ}$. microscope (SEM, FEI Quanta 200 F).

The chemical composition of all liquid samples was characterized and qualified by Agilent 7890A GC-MS (GC-MS; GC, Agilent 7890A; MS, Agilent 5975C) with a DB-5 capillary column. The $\mathrm{GC}$ was first heated to $45^{\circ} \mathrm{C}$ for $3 \mathrm{~min}$, followed by heating to $300^{\circ} \mathrm{C}$ at a rate of $10^{\circ} \mathrm{C} / \mathrm{min}$. The injection of the sample volume was $1 \mu \mathrm{L}$. The flow rate of the carrier gas (helium) was $0.6 \mathrm{~mL} / \mathrm{min}$. The ion source temperature was $230{ }^{\circ} \mathrm{C}$ for the mass selective detector. Compounds were identified by comparing the spectral data with that in the NIST Mass Spectral library. All the measurements were triplicated to ensure reproducibility.

9

The moisture content in the bio-oils was measured by a Karl Fischer (KF) compact titrator (V20 Compact Volumetric KF Titrator, Mettler-Toledo). 
1 The gas was collected in a 1L Tedlar gas bag and then offline analyzed by an INFICON 3000

2 Micro-GC (INFICON Inc., Santa Clara, CA, USA) system with a thermal conductivity detector

3 (TCD). A standard gas mixture including $\mathrm{H}_{2}, \mathrm{~N}_{2}, \mathrm{CH}_{4}, \mathrm{CO}, \mathrm{CO}_{2}, \mathrm{C}_{2} \mathrm{H}_{4}, \mathrm{C}_{2} \mathrm{H}_{6}$, and $\mathrm{C}_{3} \mathrm{H}_{6}$ was

4 quantify to calibrate the yield of non-condensable gas. The gas composition $\left(>\mathrm{C}_{4}\right)$ were either

5 not detected or negligible in this research. All the measurements were triplicated to assure 6 reproducibility.

\subsection{Experimental methods and data evaluation}

A central composite experimental design (CCD) was employed to optimize the process conditions and product yields from co-feeding of biomass with plastics in catalytic microwave pyrolysis (Table 2$)$. The catalytic temperature $\left(\mathrm{X}_{1},{ }^{\circ} \mathrm{C}\right)$ and plastics to biomass ratio $\left(\mathrm{X}_{2}\right)$ were used as independent variables. The loading of Douglas fir sawdust pellets was $20 \mathrm{~g}$ for each run, while the catalyst to reactants ratio was constantly held at 0.2 in co-feed catalytic microwave pyrolysis. In these experiments based on CCD, the mass of LDPE pellets varied from 8 to $22 \mathrm{~g}$, while the catalytic temperature ranged from 269 to $481{ }^{\circ} \mathrm{C}$. Four additional experiments were conducted as the controls (Entry $14-17$, Table 2).

The coke mass was calculated by the difference before and after catalytic process. Because of $e x$ situ catalysis, char was left in the quartz flask inside microwave over chamber; while the coke was formed on the ZSM-5 catalyst in the packed-bed catalysis reactor. The weight of gas product was determined using the following equation. 
Weight of gas = initial reactants mass - liquid mass - char mass - coke mass

2 Overall carbon yields of the liquid, gas, and solid products and carbon selectivity of a specific

3 product were calculated based on the following equations.

$$
\begin{aligned}
& \text { Carbon yield }=\frac{\text { moles of carbon in a product }}{\text { moles of carbon fed in }} \times 100 \% \\
& \text { Carbon seletivity }=\frac{\text { moles of carbon in a product }}{\text { moles of carbon in identified products }} \times 100 \%
\end{aligned}
$$

\section{$5 \quad 3$ Results and discussion}

\subsection{Catalyst characterization}

7 After the integral treatments, the BET surface area, pore surface area, and pore volume were

8 considerably improved. Besides, it was found that parent ZSM-5 fabricated by means of the 9 integrated treatments leaded to the generation of mesopores in the ZSM-5 zeolite matrix as listed in Table 3. The average pore size of the promoted ZSM-5 is $5.2 \mathrm{~nm}$, which is identical to

11 naphthalene diameter $(5.5 \mathrm{~nm})$, therefore double-ring aromatics are readily adsorbed in the 12 mesopores.[46] The overall acid amounts can be determined by the relative peak areas of the $13 \mathrm{NH}_{3}$ desorption curves; it can be seen that the acidity are comparable (Fig. 1). For the promoted 14 ZSM-5 catalyst, the maximum peak shifted to low temperatures, accompanying with a decrease 15 of strong acid sites. The decrease of strong acid sites could reduce the extent of catalytic 16 cracking of large molecules into gaseous molecules, thereby increasing the carbon yield of liquid 17 organics. However, the minimum peck was not impacted by the integrated treatments. The total 
4 For the manufacture of home-made Raney Ni catalyst, $\mathrm{Al}$ in the $\mathrm{Ni}-\mathrm{Al}$ alloy powder reacted with $5 \mathrm{NaOH}$ solution during alkali leaching. The textural properties of $\mathrm{Ni}-\mathrm{Al}$ alloy and home-made

6 Raney Ni catalyst were also demonstrated in Table 3. According to the alkali treatment, BET 7 surface area, pore surface area, and pore volume were substantially enhanced. The BET surface 8 area of home-made Raney Ni catalyst dramatically increased from 0.35 to $52.4 \mathrm{~m}^{2} / \mathrm{g}$. Moreover, 9 pore surface area and pore volume progressively went up to $35.8 \mathrm{~m}^{2} / \mathrm{g}$ and $0.034 \mathrm{~cm}^{3} / \mathrm{g}$,

acidity $\left(0.18 \mathrm{mmol} \mathrm{NH}_{3} / \mathrm{g}\right)$ of the well-promoted $\mathrm{ZSM}-5$ catalyst is a bit less than that of parent ZSM-5 due to the decrease of strong acid sites. respectively. Accordingly, the decent BET surface area of the Raney Ni catalyst contributed to the adsorption of hydrogen for the hydrogenation reaction. The average pore size of the Raney Ni catalyst is $3.8 \mathrm{~nm}$, which is suitable for the diffusion of mono-cyclic aromatics in the pores. [46]

Fig. 2 shows the XRD patterns of the Ni-Al alloy powder and home-made Raney Ni catalyst. It can be seen that the XRD patterns of $\mathrm{Ni}-\mathrm{Al}$ alloy is comprised of the two categories of $\mathrm{Ni}_{3} \mathrm{Al}_{2}$ and $\mathrm{Ni}_{3} \mathrm{Al}$ domains. As the $\mathrm{Al}$ component dissolved by 20 wt. $\% \mathrm{NaOH}$ solution, the diffractions regarding metallic $\mathrm{Ni}$ were shown as amorphous nature. It was also found that the home-made Raney Ni catalyst mainly presented diagnostic (111), (200), and (220) diffractions of fcc Ni at $2 \theta$ of $44.5,51.8$, and $76.3^{\circ}$, respectively.[47] 
3. The morphological difference between $\mathrm{Ni}-\mathrm{Al}$ alloy powder and home-made Raney Ni catalyst are readily visible from Fig. 3 (A) and (B). The home-made Raney Ni catalyst is constituted by the typical fractured and angular particles, which is identical to other research;[48] whereas NiAl alloy powder only had the intact metallic structure. In addition, it is observed that the small particles of home-made Raney Ni catalyst are dispersive, reaffirming the high BET surface.

\subsection{Product yield distributions from co-feed catalytic microwave pyrolysis}

The product yield distribution from co-feeding of lignocellulosic biomass with plastics in the catalytic microwave pyrolysis are summarized on the basis of catalytic temperature and plastics to biomass mass ratio in Table 2. It was found that the mass yields of bio-oil and gas were considerably impacted by the two variables in the range from 30.57 to $47.00 \mathrm{wt} \%$ versus 36.18 to $49.56 \mathrm{wt} \%$, respectively. Without the addition of plastics, the yield of bio-oil derived from catalytic microwave pyrolysis of lignocellulosic biomass alone was much less than that of biooils from the co-feed catalytic microwave pyrolysis of biomass with plastics. The water content in the bio-oil varied from approximately 10 to $30 \mathrm{wt} \%$ depending upon the reaction conditions. Without the introduction of plastics in the catalytic microwave pyrolysis, the water content could reach $30 \mathrm{wt} \%$, while the water content from the co-feed catalytic microwave pyrolysis was $\sim 10$ - $20 \mathrm{wt} \%$. When the optimal condition of plastics to biomass ratio for minimizing the water content was executed at 1.45 , the water content was declined to $9.54 \mathrm{wt} \%$. It is evidenced that plastics-derived olefins could react with furan compounds from the decomposition of cellulose and hemicellulose to generate aromatics through Diels-Alder reactions followed by dehydration $[24,49]$. As a result, if the extent of dehydration reaction was enhanced in the co-feed catalytic 
microwave pyrolysis; the increasing trend of water content in the bio-oil was observed. In addition, the yield of olefins produced via catalytic cracking and subsequent cyclization for the production of aromatics could also be influenced by the two independent variables.[45] Accordingly, the catalytic temperature and plastics to biomass ratio play an essential role bio-oil and gas yields. The maximum bio-oil yield was observed at the catalytic temperature of $200{ }^{\circ} \mathrm{C}$ with the plastics to biomass ratio of 0.75 ; while the maximum gas yield was found at $500{ }^{\circ} \mathrm{C}$ with the ratio of 0.75 .

Owing to the ex-situ catalysis, char derived from microwave-assist co-pyrolysis and coke from catalytic step can be distinctly separated. It was also found that catalytic temperature and plastics to biomass ratio affected both mass yields of char and coke as well. The char yield was in the range from $9.09 \mathrm{wt} \%$ at the highest plastics to biomass ratio of 1.45 to $21.69 \mathrm{wt} \%$ without the introduction of plastics. Thus the char residue from the catalytic microwave co-pyrolysis of lignocellulosic biomass with plastics was lower than that from biomass alone. These results indicate that co-feeding of biomass with plastics in the catalytic microwave pyrolysis was discerned to have a pronounced impact at the char formation. It is mainly attributed to the fact that with in the surrounding of hydrogen from the direct decomposition of plastics, the polymerization reactions of oxygenates were partially inhibited for the char formation $[26,50]$. In addition, lignin as the main component of lignocellulosic biomass (Table S1) generated reactive free radicals upon pyrolysis [51]. These free radicals were relatively unstable and could readily polymerize during pyrolysis; therefore, the free radicals became strong hydrogen acceptors [52]. Hydrogen evolved from plastics could be likely absorbed by free radicals, and the polymerization and cross-linking reactions to form char were thus suppressed [21]. As a result, 
the formation of char was strongly reduced when biomass was co-pyrolyzed with plastics. From other studies reported elsewhere, the HHV of the resulting char could be obviously improved in the co-pyrolysis of biomass and plastics as well. [50, 53]

Since the coke deposited on the catalyst could cause the deactivation of zeolite-based catalysts,[40] the co-feed catalytic microwave pyrolysis should aid in reducing coke formation and extending catalyst lifetime. The coke yield with regard to catalytic temperature and plastics to biomass ratio is also outlined in Table 2, ranging from 1.02 to $3.85 \mathrm{wt} \%$. As expected, the coke formation was notably affected by the co-feeding of plastics. It can be seen that the highest coke yield was obtained without the addition of plastics. The catalyst coke was attenuated as the plastics was co-fed, declining to $\sim 1 \mathrm{wt} \%$. It has been reaffirmed that in the presence of the wellpromoted ZSM-5 catalyst, the resultant furan compounds could react with plastics-derived olefins via Diels-Alder reactions rather than polymerization reactions, thereby reducing the coke formation [8, 9]. Furthermore, the hydrogen-deficient oxygenates from the thermal decomposition of cellulose and hemicellulose could accept the hydrogen from plastics degradation or olefins oligomerization, resulting in the decrease of coke formation [26]. For most phenolic compounds from the thermal degradation of lignin were too large to enter zeolite pores [54]; these phenolic compounds could easily deposit on the catalyst surface and further polymerize to form coke [21]. Conversely, these phenolic compounds could abstract hydrogen atoms from evolutions of plastics during the co-feed catalytic microwave pyrolysis. Hence these phenolic compounds were stabilized and the polymerization reaction for the generation of coke could be inhibited. In the ANOVA analysis based on CCD, the $P$-value from the four models (bio-oil, gas, char, and coke yield) are all smaller than 0.05 , implying that these models are 
appropriate to present the relationships between all product yields and the two independent variables as sketched in Fig. S3 - S6. The coefficient of determination $\left(R^{2}\right)$ of these models are all more than 0.95 , which evidences that these models are favorable to mimic the relationships. Accordingly, these models can be employed to predict the maximum product yields regarding the two independent variables.

\subsection{The effect of catalytic temperature on the co-feed catalytic microwave pyrolysis}

As reported in our previous work, catalyst temperature played a vital in the product distribution $[40,44]$. In this case, catalytic temperature was also used to investigate the product distribution and evaluate the qualities of liquid organics. Representative results of product distribution during co-feed catalytic microwave pyrolysis in light of catalytic temperature are shown in Fig. 4. As expected, the carbon yield of char did not significantly vary alongside the changes of catalytic temperature because of the ex-situ catalysis. Increasing the catalyst temperature during co-feed catalytic microwave pyrolysis lowered the carbon yield of catalyst coke. It was noted that direct degradation of plastics could produce a large amount of long-chained waxes.[21] However, these waxes with long carbon chins could be readily adsorbed on the catalytic surface at the low catalytic temperatures, forming the waxy coke that finally enclosed the whole catalyst. Meanwhile, phenolic compounds could also be abstracted on the catalyst and stepwise polymerize to yield coke at the low catalytic temperatures [23]. When the elevated catalytic temperatures implemented, catalytic cracking of waxes and phenolic compounds was favored, generating lighter compounds which were more easily accessible to enter the mesopores. Consequently, the carbon yield of coke was reduced at the expense of large molecules as the 
3 Since the degradation of plastics to generate olefins is an endothermic reaction, the yield of

4 olefins should go up as the catalytic temperature increased at the tested region [15]. The

5 increased yield of olefins could strengthen the Diels-Alder reaction, contributing to the increase

6 of aromatics [23]. Nonetheless, an excessively high catalytic temperature can favor reverse

7 Diels-Alder reactions to form light olefins instead of aromatics [8, 26]. It was also manifested

8 that increasing catalytic temperature improved the decomposition of biomass-derived oxygenates

9 into small molecular, preventing the formation of liquid aromatics $[6,8]$. As a result, the carbon

10 yield of liquid organics gradually decreased due to the enhanced catalytic cracking reactions in

11 the catalytic temperature region. On the contrary, the carbon yield of gas monotonically

12 promoted at the expense of liquid organics degradation as the catalytic temperature went up,

13 reaching $46.22 \%$ at $500{ }^{\circ} \mathrm{C}$.

14
Table 4 gives a detailed carbon yield and selectivity on the basis of catalytic temperature. In comparison with the liquid organics obtained by co-feed catalytic microwave pyrolysis at $200{ }^{\circ} \mathrm{C}$, the aliphatic olefins and oxygenates were the primary species in the liquid organics. This result indicates that such low catalytic temperature could not favor the zeolite-catalyzed reactions to form aromatics. As the catalytic temperature was elevated to $375{ }^{\circ} \mathrm{C}$, the carbon selectivity toward aromatic hydrocarbons remarkably increased. There was still a low amount of aliphatic hydrocarbons in the liquid organics (at $375^{\circ} \mathrm{C}$ ). Besides, there was a trace amount of aromatic oxygenates (such as phenol) existing in the liquid organics, whose content was much lower than 
those from catalytic microwave pyrolysis of biomass alone under the same conditions [44]. It is likely that plastics-derived olefins participated in the Diels-Alder reaction with furan compounds followed by dehydration reaction to expel these oxygenates. Aromatic hydrocarbons obtained were predominantly made up of toluene, xylenes, trimethylbenzenes, naphthalene, and their derivatives. It was obtained that the carbon selectivity toward toluene and xylenes ranged from 10.72 to $18.42 \%$ and 18.78 to $27.41 \%$, respectively; whilst the carbon selectivity toward trimethylbenzene decreased from 17.13 to $9.01 \%$ as catalytic temperature went up from 375 to $500{ }^{\circ} \mathrm{C}$. These outcomes evidence that the dealkylation reaction of aromatics was also promoted

9 at the elevated catalytic temperature, leading to increased amounts of simple aromatics.[15, 21] It is noted that a trace amount of oxygenates was obtained at the catalytic temperature of $500{ }^{\circ} \mathrm{C}$. It could be inferred from these results that under such condition, hydrogen transfer reactions could enhance the cracking of the side chains substituted on phenyl to yield aromatic hydrocarbons.[54] It is also possible that the stabilized phenolic compounds could be directly deoxygenated via demethoxylation followed by dehydroxylation to obtain simple aromatic hydrocarbons.[21] To make the precursors match the jet fuels, these results suggest that the aromatic hydrocarbons obtained at catalytic temperature of $375{ }^{\circ} \mathrm{C}$ are more desirable.

Non-condensable gas was another major co-product from co-feeding of biomass with plastics in the catalytic microwave pyrolysis. The composition of gaseous fraction regarding catalytic temperature is also listed in Table 4. Because of the co-feeding of biomass with plastics in the catalytic microwave pyrolysis, oxygen content was expelled from furan compounds by reacting with olefins through Diels-Alder reaction followed by dehydration reaction with the water as product, instead of decarbonylation and decarboxylation reactions [7, 8, 49]. Thus the carbon 
yields of $\mathrm{CO}$ and $\mathrm{CO}_{2}$ are very low. However, it was observed that the carbon yields of $\mathrm{CO}$ increased as the catalytic temperature went up from 375 to $500{ }^{\circ} \mathrm{C}$. It is discerned that higher catalytic temperature had a negative impact at the Diels-Alder reaction [21], resulting in rejecting oxygen content from furans by decarbonylation reaction. It is noteworthy that the carbon yield of ethylene dramatically increased as the catalytic temperature went up to $500{ }^{\circ} \mathrm{C}$. These results indicate that increasing catalytic temperature favored the catalytic cracking of long-chain waxes into light olefins. In addition, elevated catalytic temperature also prevented the aromatization reaction of light olefins to form aromatic hydrocarbons. [10, 15]

\subsection{The effect of plastics to biomass ratio on the co-feed catalytic microwave pyrolysis}

It was manifested that the optimal condition to obtain aromatic hydrocarbons lumped in the jet fuels range was set at $375^{\circ} \mathrm{C}$. Since lignocellulosic biomass is more abundant and cheaper than waste plastics, it is desired to enhance the mass ratio of cellulosic biomass in the co-reactants if this cannot decrease the carbon yield of liquid organics substantially. In order to investigate the effect of plastics proportion in the co-feed catalytic microwave pyrolysis, the overall carbon yield of product distribution at $375{ }^{\circ} \mathrm{C}$ with respect to plastics to biomass ratio is depicted in Fig. 5. It can be seen that the carbon yield of liquid organics increased nonlinearly with the augment of plastics to biomass ratio. Comparing with liquid organics without using plastics as the coreactant, the liquid carbon yield rapidly increased as the ratio went up to 0.75 . This increasing tendency indicates that there was a positive synergy between the two reactants. Furthermore, the substantial trend was attributed to the fact that large amounts of olefins with high $\mathrm{H} / \mathrm{C}_{\text {eff }}$ ratio reacted with furan compounds by the Diels-Alder reaction to enhance liquid organics yield [55, 
56]. According to our previous study [45], the highest yield (above 30\%) of liquid organics from catalytic microwave pyrolysis of LDPE alone was much less than the yield of liquid organics from co-feed catalytic microwave pyrolysis. Yet as the plastics to biomass ratio went up from 0.75 to 1.45 , the carbon yield of liquid organics slightly augmented. Thus, this slight tendency suggests that the co-feeding of biomass and plastics in catalytic microwave pyrolysis through the Diels-Alder reaction for the enhancement of liquid organics yield could not take place to a larger extent or could even reach the ceiling.

Both the carbon yields of char and coke gradually declined with an increasing plastics to biomass ratio as shown in Fig. 5. The carbon yields dramatically first decreased as the plastics to biomass ratio went up to 0.75 ; whereas they represent slight augmented trends as the ratio started 0.75 to 1.45. These could be explained by the fact that there was a significantly synergistic effect between the two feedstock in the ratio range from 0 to 0.75 ; whereas the synergy could not be further appreciably enhanced as more plastics were employed. Hence, there exists an optimal plastics to biomass ratio $(0.75)$ for the Diels-Alder reaction in co-feed catalytic microwave pyrolysis if considering high value of waste plastics. As stated above, the Diels-Alder reaction could not be further enhanced at the high ratios $(0.75-1.45)$, thus the light olefins derived from the catalytic cracking improved the carbon yield of non-condensable gas.

Table 5 lists how the carbon selectivity toward aromatics changes with the addition of plastics. In general, the carbon selectivity toward monocyclic aromatics went up as the plastics to biomass ratio increased; whist the carbon selectivity toward polycyclic aromatics gradually declined. For 
1 instance, the maximum carbon selectivity of aromatic hydrocarbons was xylenes, which

2 increased appreciably from 4.28 to $21.33 \%$. As such, the second highest carbon selectivity of

3 aromatics was trimethylbenzene, displaying the same tendency as xylenes from 8.32 to $16.25 \%$.

4 By contrast, the carbon selectivity toward polycyclic aromatics experienced a remarkable drop

5 with the increasing plastics to biomass ratio. It is due to the reason that Diels-Alder reaction

6 between aromatics and light olefins, or hydrogen could suppress the polymerization reaction of

7 monocyclic aromatics to generate polycyclic aromatics [21, 22, 26]. Meanwhile, light olefins

8 alone were prone to the formation of monocyclic aromatic hydrocarbons by using ZSM-5

9 catalyst in the catalytic microwave pyrolysis. [45] In this regard, the aromatics with the carbon

10 number in the jet fuel range were preferred from the experiment conducted at the low plastics to

11 biomass ratio.

13 The gaseous composition with regard to plastics to biomass ratio is also illustrated in Table 5.

14 The carbon yield of methane retains at around $12 \%$ with the maximum yield at the ratio of 0.75 ,

15 whereas, the carbon yield of both $\mathrm{CO}$ and $\mathrm{CO}_{2}$ decreased with the increase of plastics to biomass

16 ratio. It is mainly attributed to the Diels-Alder reaction that was accelerated between biomass

17 and plastics, instead of the decarbonylation and decarboxylation reaction. Unlike the $\mathrm{CO}$ and

$18 \mathrm{CO}_{2}$, the carbon yield of ethylene was found to augment in the most pronounced way from 5.32

19 to $56.34 \%$. These outcomes verifies that the introduction of plastics in the catalytic microwave

20 pyrolysis enhanced the $\mathrm{H} / \mathrm{C}_{\text {eff }}$ ratio; and biomass-derived oxygenates was futher transformed into

21 more aromatics and olefins. Besides, biomass-derived oxygenates could promote the degradation

22 of large molecules to light olefins in the co-feed catalytic pyrolysis.[15] 
Irrespective of conversion extent of co-feed catalytic microwave pyrolysis, an appreciable

4 reaction condition was employed to determine the process robustness and reuse of the well-

5 promoted ZSM-5 catalyst. That is because the catalyst recyclability is of essential importance for

6 a heterogeneous process [57]. Thus, the spent catalyst was regenerated to evaluate the

7 recyclability in the co-feed catalytic microwave pyrolysis. The conversions were all conducted at

8 the catalytic temperature of $375{ }^{\circ} \mathrm{C}$ with the plastics to biomass ratio of 0.75 as presented in Fig.

9 6. Since the co-feeding of biomass and plastics in the microwave reactor was pyrolyzed under

10 the same condition, the carbon yield of char did not significantly change along with the recycle

11 times of the catalyst. However, the carbon yield of liquid organics was dramatically affected by

12 the catalyst recycle times, showing a slight decrease with the increase of recycle times. This

13 result verifies that although coke deposited on the catalyst surface could be removed, the

14 mesopores were permanently blocked by the coke, triggering the loss of active sites. Thus the

15 Diels-Alder reaction between the biomass and plastics for the improvement of liquid organics

16 yield was inhibited as well. Interestingly, the carbon yield of coke first decreased then increased.

17 The decreased carbon yield of coke was possibly due to the fact that the Diels-Alder

18 cycloaddition was inhibited in the pores; yet the catalytic cracking still took place on the surface

19 of the catalyst active sites, which could mitigate the polymerization to form coke. For the

20 increased carbon yields, the active sites on the catalyst surface were also be deactivated, leading

21 to the waxes absorbed on the catalyst that could not be cracked. According to the trend of gas

22 yield, it is reaffirmed that the active sites inside the catalyst pores was first deactivated by the

23 pore blockage, the active sites on the catalyst surface were subsequently inhibited with the 
increasing recycle times of the catalyst.

3 Table 6 describes the detailed carbon yields and product carbon selectivity with respect to

4 catalyst reused times. It was found that carbon selectivity toward all aromatics species decreased

5 with the increasing reused times. Nonetheless, a large amount of aliphatic hydrocarbons were

6 detected in the liquid organics. The results indicate that the aromatization and oligomerization of

7 olefins to form aromatics were suppressed due to catalyst pores blockages; the Diels-Alder

8 cycloaddition between the co-reactants for improving aromatics carbon selectivity was thus

9 inhibited. It is noticed that both the carbon selectivity of $\mathrm{CO}$ and $\mathrm{CO}_{2}$ first decreased and then

10 went up; whilst the carbon selectivity of $\mathrm{C}_{2} \mathrm{H}_{4}$ showed an inverse tendency, first increasing and

11 then decreasing from the fresh to the fifth usage. It is manifested that the catalytic cracking of

12 both waxes and oxygenates occurred individually on the catalyst surface, forming these relevant

13 gas in the first phase (from the fresh use to the third use). However, as the active sites was

14 deactivated on the surface from the third use to the fifth use, the catalytic cracking of waxes and

15 oxygenates was suppressed as well.

16

173.6 Hydrogenation of liquid organics derived from co-feed catalytic microwave pyrolysis for jet

18 fuels

19 Given the Raney Ni catalyst could be poisoned by water in the hydrogenation process [36], the

20 water in the raw bio-oil samples should be removed. Since $n$-heptane severed as the vital

21 medium in the hydrogenation process [35], two phase of mixed bio-oil samples was separated by

22 the optimum solvent ( $n$-heptane). The liquid organics mixed with $n$-heptane from every samples 
were separated and weighed to measure the loss of organics. $~ 94 \mathrm{wt} \%$ of liquid organics were existing in the organic phase by extracting all samples. Since liquid organics produced from catalytic microwave co-pyrolysis (at $375{ }^{\circ} \mathrm{C}$ with the plastics to biomass ratio of 0.75 ) were principally comprised of aromatics from $\mathrm{C}_{8}-\mathrm{C}_{16}$, these aromatics are viewed as precursors for civilian jet fuels. In addition, the run that obtained the maximum liquid carbon yield (at $375{ }^{\circ} \mathrm{C}$ with the ratio of 1.45) and the run that gained the liquid organics within lowest oxygenated content (at $500{ }^{\circ} \mathrm{C}$ with the ratio of 0.75 ) were also hydrogenated for the optimization of jet fuels. As reported, naphthalene in $n$-heptane was completely transformed into saturated decalin [35], thus the mass ratio of reactant to $n$-heptane was set at 1:7 in terms of adding certain mass of $n$-heptane.

The product distribution and carbon selectivity toward main alkanes from the hydrogenation of diverse raw organics are elucidated in Table 7 . After all the reactions, more than 99 vol\% of unreacted hydrogen was detected, indicating that the hydrogenation reactions were not implemented under hydrogen-starved conditions. The overall carbon yields of these hydrogenated organics (R-1, R-2, and R-3) with respect to the co-reactants of biomass and plastics were $38.51,32.92$, and $40.53 \%$, respectively. These carbon yields of hydrogenated organics were much higher than that in our previous research [35]. Compared with the overall product distribution of hydrogenated R-1 and R-3, the result regarding the production of cycloalkanes from hydrogenated R-2 was more superior to the others under the same condition. It was observed that $\sim 90 \%$ selectivity toward cycloalkanes was achieved from hydrogenated R-2. The high amounts of high-density cycloalkanes can be potentially used as the replacement of high-density jet fuels (e.g., JP-10 and RJ-5). For the production of aliphatic alkanes, 
hydrogenated R-3 obtained the maximum amount, which is close to $30 \%$. In this sense, the

2 contents of hydrogenated R-3 were identical to that in JP-5 navy fuel $(31.23 \%$ of aliphatic

3 alkanes, $53.06 \%$ of cycloalkanes, and $15 \%$ of aromatic hydrocarbons) [58]. The total amounts of

4 hydro-aromatic hydrocarbons and aromatic hydrocarbons were comparable in all hydrogenated

5 organics, whose amounts satisfy the regulations (less than 15\%) of current jet fuels. A trace

6 mount of other compounds were detected in all hydrogenated organics. Likewise, lower than 1

7 vol\% of small hydrocarbons (e.g., methane, ethane, and propane) were achieved under the low-

8 severity condition, reaffirming that there was almost no carbon loss in the hydrogenation system.

It was discerned that hydrogenated R-3 attained the maximum carbon selectivity toward monocyclic alkanes, except dimethylcyclohexane. Dimethylcyclohexane derived from the hydrocycloaddition of xylenes presented the highest carbon selectivity in hydrogenated R-2. That is because the dealkylation reaction was facilitated at the high catalytic temperature $\left(500{ }^{\circ} \mathrm{C}\right)$,

14 giving rise to high yield of xylenes. It is also noticeable that the maximum carbon selectivity

15 toward aliphatic alkanes was attained in hydrogenated R-3 and none of unsaturated aliphatic 16 hydrocarbons was found, suggesting that these high amounts of unsaturated aliphatic 17 hydrocarbons were completely transformed into saturated aliphatic hydrocarbons under the mild 18 reaction condition. Of the three raw organics for hydrogenation, the hydrogenated organics 19 derived from R-1 obtained the highest amounts of saturated alkanes lumped in the jet fuel range. 20 With the consideration of both reaction condition and product distribution, the raw organics $(\mathrm{R}-1)$ 21 is the optimal source to manufacture renewable jet fuels with high carbon yield. 
2 fuels

3 These observations are the key support to show the reaction route for the conversion regarding

4 co-feeding of lignocellulosic biomass with plastics into jet fuels. The resultant observation of the

5 hydrogenated organics is manifested that the reaction pathway was much more complicated than

6 those proposed previously $[9,14,26]$. Based on the quantified products in this research, related

7 results from catalytic microwave-induce degradation of LDPE alone [45], and lignocellulosic

8 biomass to jet fuel through combined catalytic conversions [40, 59]; the overall reactions

9 network (co-feed catalytic pyrolysis and hydrogenation process) is outlined in Fig. 7. For the

10 dominant route in lignocellulosic biomass, cellulose went through a sequence of dehydration,

11 decarbonylation, and decarboxylation to generate furan compounds in the thermal degradation

$12[17,40]$. As such, it was evidenced that hemicellulose was likely to depolymerized into furan

13 compounds [59], which was comparable with the result of cellulose degradation [40]. Unlike

14 cellulose and hemicellulose, lignin in lignocellulosic biomass was primarily decomposed into

15 phenolic compounds. As for the degradation of plastics in another route, thermal degradation of

16 plastics usually occurred through two mechanisms (random scission and chain-end scission) [60,

17 61]. The two abovementioned mechanisms took place simultaneously, generating free radicals

18 together with the long carbon chains [62]. At the same time, the radical fragments could be

19 transformed into straight chain hydrocarbons via hydrogen transfer reactions [62]. The hydrogen

20 from the thermal degradation of plastics was provided for biomass-derived oxygenates that acted

21 as the strong acceptor, suppressing the char formation. 
The waxes with large molecule weigh from the thermal degradation of plastics subsequently

2 underwent catalytic cracking over ZSM-5 through two carbocationic mechanisms, giving rise to

3 light olefins $[60,63]$. These olefins thereafter reacted with furan compounds by the Diels-Alder

4 reaction followed by the dehydration reaction to form aromatic hydrocarbons. Meanwhile, these

5 plastics-derived olefins could individually subject to cyclization, aromatization, and

6 oligomerization reactions to obtain aromatic hydrocarbons [21]. Likewise, these furan

7 compounds could individually go through decarbonylation, aromatization, and oligomerization

8 reactions inside the mesopores of well-promoted ZSM-5 to form aromatic hydrocarbons as well.

9 As previously reported, it is confirmed that the interaction via the hydrocarbon pool mechanism

10 could be found in addition to Diels-Alder reaction [9]. Since the carbon yield of liquid organics

11 significantly increased and the coke yield dramatically decreased, the Diels-Alder reaction was

12 the dominant reaction pathway during the co-feed catalytic pyrolysis in comparison with the

13 hydrocarbon pool mechanism. In addition, phenolic compounds were individually converted into

14 aromatic hydrocarbons over ZSM-5 catalyst by the dehydration, cracking, and oligomerization

15 reactions $[17,64]$.

17 The liquid organics resulting from the co-feed catalytic microwave pyrolysis mostly gave rise to

18 a mixture of aromatic and aliphatic hydrocarbons. As hydrogenation of unsaturated hydrocarbons

19 primarily occurs in bio-oil hydrotreatment by using metal catalysts under mild reaction

20 conditions [65], and Raney-Ni catalysts in the hydrotreating process are in favor of catalyzing

21 the hydrogenation reactions due to the porous structure of active nickel [66]; hence, it was shown

22 that the hydrogenation of aliphatic olefins was the first step to take place in the hydrogenation

23 system [67]. The aromatic hydrocarbons were stepwise hydrogenated into cyclic alkanes or 
hydro-aromatic hydrocarbons through hydro-cycloaddition reactions in the presence of home-

2 made Raney Ni catalyst. Furthermore, the hydroisomerization reaction could took place among

3 the dimethylcyclohexanes; and a trace volume of small hydrocarbons was also generated by

4 hydrocracking reactions. As a result, these hydrogenated organics can be potentially used as

5 alternatives of civilian jet fuels or additives to manufacture desired jet fuels by mixing with other

6 hydrocarbons.

\section{Conclusions}

9 In summary, the microwave-induced co-pyrolysis followed by hydrogenation process is a 10 profound route for the improved carbon yield of jet fuel range alkanes from co-feeding of 11 lignocellulosic biomass with plastics. Two variables (catalytic temperature and plastics to 12 biomass ratio) were evaluated in the co-feed catalytic microwave pyrolysis to improve the 13 carbon yield of aromatics with the carbon number in the jet fuel range. The results showed that 14 there was a positive synergy between lignocellulosic biomass and plastics in the co-feed catalytic 15 microwave pyrolysis, which could significantly improve the carbon yield of liquid organics and 16 suppress the formations of char and coke. Although the optimal condition to maximize the 17 carbon yield $(42.66 \%)$ of liquid organics in the step of co-feed catalytic microwave pyrolysis 18 was at $375{ }^{\circ} \mathrm{C}$ with the plastics to biomass ratio of 1.45 , the compounds with low carbon 19 numbers (e.g. toluene) was also obtained, which cannot meet the specifications of jet fuels. Yet 20 the chemical compounds of liquid organics with high carbon yield (40.54\%), which was derived 21 from the reaction conducted at $375{ }^{\circ} \mathrm{C}$ with the ratio of 0.75 primarily satisfy the specifications 22 in the jet fuel range. 
1
9

After the hydrogenation process by using home-made Raney $\mathrm{Ni}$ as the catalyst under a lowseverity condition, the overall carbon yield of three hydrogenated organics (R-1, R-2, and R-3) regarding raw lignocellulosic biomass and plastics were $38.51,32.92$, and $40.53 \%$, respectively. Given the overall carbon yield and product distribution, the raw organics conducted at the catalytic temperature of $375{ }^{\circ} \mathrm{C}$ with the biomass to plastics ratio of 0.75 in the co-feed catalytic microwave pyrolysis is more suitable for the production of renewable alkanes for advanced jet fuels (especially for high-density jet fuels). Herein, 90\% selectivity toward jet fuel range alkanes was obtained from such raw organics, and up to $75 \%$ selectivity belongs to high-density cycloalkanes. It is more likely that in the future these alkanes can be potentially used as additives or replacements for jet fuels.

\section{ACKNOWLEDGMENTS}

This study was supported partially by the Agriculture and Food Research Initiative of National Institute of Food and Agriculture, United States Department of Agriculture (Award Number: 2015-67021-22911; Award Number: 2016-67021-24533), WSU Office of Commercialization and the Offices of the President and Provost in conjunction with the Vice President of Research, the Washington Research foundation, and Chinese Scholarship Council. We are grateful to Dr. Aftab Ahamed and Dr. Xin Li for their assistance with GCMS measurements. 


\section{References}

[1] Kunkes EL, Simonetti DA, West RM, Serrano-Ruiz JC, Gärtner CA, Dumesic JA. Catalytic Conversion of Biomass to Monofunctional Hydrocarbons and Targeted Liquid-Fuel Classes. Science. 2008;322:41721.

[2] Kim SK, Han JY, Lee H-s, Yum T, Kim Y, Kim J. Production of renewable diesel via catalytic deoxygenation of natural triglycerides: Comprehensive understanding of reaction intermediates and hydrocarbons. Appl. Energy. 2014;116:199-205.

[3] Atsonios K, Kougioumtzis M-A, D. Panopoulos K, Kakaras E. Alternative thermochemical routes for aviation biofuels via alcohols synthesis: Process modeling, techno-economic assessment and comparison. Appl. Energy. 2015;138:346-66.

[4] Tungal R, Shende RV. Hydrothermal liquefaction of pinewood (Pinus ponderosa) for H2, biocrude and bio-oil generation. Appl. Energy. 2014;134:401-12.

[5] Srirangan K, Akawi L, Moo-Young M, Chou CP. Towards sustainable production of clean energy carriers from biomass resources. Appl. Energy. 2012;100:172-86.

[6] Carlson TR, Cheng Y-T, Jae J, Huber GW. Production of green aromatics and olefins by catalytic fast pyrolysis of wood sawdust. Energy Environ Sci. 2011;4:145-61.

[7] Cheng YT, Jae J, Shi J, Fan W, Huber GW. Production of renewable aromatic compounds by catalytic fast pyrolysis of lignocellulosic biomass with bifunctional Ga/ZSM-5 catalysts. Angewandte Chemie. 2012;51:1387-90.

[8] Cheng Y-T, Huber GW. Production of targeted aromatics by using Diels-Alder classes of reactions with furans and olefins over ZSM-5. Green Chem. 2012;14:3114.

[9] Li X, Zhang H, Li J, Su L, Zuo J, Komarneni S, et al. Improving the aromatic production in catalytic fast pyrolysis of cellulose by co-feeding low-density polyethylene. Appl. Catal., A. 2013;455:114-21.

[10] Li J, Yu Y, Li X, Wang W, Yu G, Deng S, et al. Maximizing carbon efficiency of petrochemical production from catalytic co-pyrolysis of biomass and plastics using gallium-containing MFI zeolites. Appl. Catal., B. 2015;172-173:154-64.

[11] Wang T, Qiu S, Weng Y, Chen L, Liu Q, Long J, et al. Liquid fuel production by aqueous phase catalytic transformation of biomass for aviation. Appl. Energy. 2015;160:329-35.

[12] Carlson TR, Jae J, Lin Y-C, Tompsett GA, Huber GW. Catalytic fast pyrolysis of glucose with HZSM5 The combined homogeneous and heterogeneous reactions. J. Catal. 2010;270:110-24.

[13] Shen $Y$, Zhao $P$, Shao Q, Takahashi F, Yoshikawa K. In situ catalytic conversion of tar using rice husk char/ash supported nickel-iron catalysts for biomass pyrolytic gasification combined with the mixingsimulation in fluidized-bed gasifier. Appl. Energy. 2015;160:808-19.

[14] Dorado C, Mullen CA, Boateng AA. H-ZSM5 Catalyzed Co-Pyrolysis of Biomass and Plastics. ACS Sustainable Chemistry \& Engineering. 2014;2:301-11.

[15] Zhang H, Nie J, Xiao R, Jin B, Dong C, Xiao G. Catalytic Co-pyrolysis of Biomass and Different Plastics (Polyethylene, Polypropylene, and Polystyrene) To Improve Hydrocarbon Yield in a FluidizedBed Reactor. Energy Fuels. 2014;28:1940-7.

40 [16] Liu C, Wang H, Karim AM, Sun J, Wang Y. Catalytic fast pyrolysis of lignocellulosic biomass. Chem. 41 Soc. Rev. 2014;43:7594-623.

42 [17] Wang K, Kim KH, Brown RC. Catalytic pyrolysis of individual components of lignocellulosic biomass. 43 Green Chem. 2014;16:727. 
[18] Yao W, Li J, Feng Y, Wang W, Zhang X, Chen Q, et al. Thermally stable phosphorus and nickel modified ZSM-5 zeolites for catalytic co-pyrolysis of biomass and plastics. RSC Adv. 2015;5:30485-94.

[19] Zhang $Y, B i P$, Wang J, Jiang $P, W u X, X u e ~ H$, et al. Production of jet and diesel biofuels from renewable lignocellulosic biomass. Appl. Energy. 2015;150:128-37.

[20] Zhang X, Wang T, Ma L, Zhang Q, Huang X, Yu Y. Production of cyclohexane from lignin degradation compounds over Ni/ZrO2-SiO2 catalysts. Appl. Energy. 2013;112:533-8.

[21] Xue Y, Kelkar A, Bai X. Catalytic co-pyrolysis of biomass and polyethylene in a tandem micropyrolyzer. Fuel. 2016;166:227-36.

[22] Dorado C, Mullen CA, Boateng AA. Origin of carbon in aromatic and olefin products derived from HZSM-5 catalyzed co-pyrolysis of cellulose and plastics via isotopic labeling. Appl. Catal., B. 2015;162:338-45.

[23] Zhang H, Xiao R, Nie J, Jin B, Shao S, Xiao G. Catalytic pyrolysis of black-liquor lignin by co-feeding with different plastics in a fluidized bed reactor. Bioresour. Technol. 2015;192:68-74.

[24] Nikbin N, Do PT, Caratzoulas S, Lobo RF, Dauenhauer PJ, Vlachos DG. A DFT study of the acidcatalyzed conversion of 2,5-dimethylfuran and ethylene to p-xylene. J. Catal. 2013;297:35-43.

[25] Green SK, Patet RE, Nikbin N, Williams CL, Chang C-C, Yu J, et al. Diels-Alder cycloaddition of 2methylfuran and ethylene for renewable toluene. Appl. Catal., B. 2016;180:487-96.

[26] Li X, Li J, Zhou G, Feng Y, Wang Y, Yu G, et al. Enhancing the production of renewable petrochemicals by co-feeding of biomass with plastics in catalytic fast pyrolysis with ZSM-5 zeolites. Appl. Catal., A. 2014;481:173-82.

[27] Miskolczi N, Bartha L, Deák G, Jóver B. Thermal degradation of municipal plastic waste for production of fuel-like hydrocarbons. Polym. Degrad. Stab. 2004;86:357-66.

[28] Xing R, Subrahmanyam AV, Olcay H, Qi W, van Walsum GP, Pendse H, et al. Production of jet and diesel fuel range alkanes from waste hemicellulose-derived aqueous solutions. Green Chem. 2010;12:1933.

[29] Huber GW, Iborra S, Corma A. Synthesis of Transportation Fuels from Biomass: Chemistry, Catalysts, and Engineering. Chem Rev. 2006;106:4044-98.

[30] Balster LM, Corporan E, DeWitt MJ, Edwards JT, Ervin JS, Graham JL, et al. Development of an advanced, thermally stable, coal-based jet fuel. Fuel Process. Technol. 2008;89:364-78.

[31] Meylemans HA, Quintana RL, Goldsmith BR, Harvey BG. Solvent-free conversion of linalool to methylcyclopentadiene dimers: a route to renewable high-density fuels. ChemSusChem. 2011;4:465-9.

[32] Zhao C, Kou Y, Lemonidou AA, Li X, Lercher JA. Highly selective catalytic conversion of phenolic bio-oil to alkanes. Angewandte Chemie. 2009;48:3987-90.

[33] Leung DYC, Wu X, Leung MKH. A review on biodiesel production using catalyzed transesterification. Appl. Energy. 2010;87:1083-95.

[34] Turner JWG, Pearson RJ, Dekker E, losefa B, Johansson K, ac Bergström K. Extending the role of alcohols as transport fuels using iso-stoichiometric ternary blends of gasoline, ethanol and methanol. Appl. Energy. 2013;102:72-86.

[35] Zhang X, Lei H, Zhu L, Wu J, Chen S. From lignocellulosic biomass to renewable cycloalkanes for jet fuels. Green Chem. 2015;17:4736-47.

[36] Zhang X, Lei H, Zhu L, Wei Y, Liu Y, Yadavalli G, et al. Production of renewable jet fuel range alkanes and aromatics via integrated catalytic processes of intact biomass. Fuel. 2015;160:375-85.

[37] Zhang X, Lei H. Synthesis of high-density jet fuel from plastics via catalytically integral processes. 
RSC Adv. 2016;6:6154-63.

[38] Chuck CJ, Donnelly J. The compatibility of potential bioderived fuels with Jet A-1 aviation kerosene. Appl. Energy. 2014;118:83-91.

[39] Iliopoulou EF, Antonakou EV, Karakoulia SA, Vasalos IA, Lappas AA, Triantafyllidis KS. Catalytic conversion of biomass pyrolysis products by mesoporous materials: Effect of steam stability and acidity of Al-MCM-41 catalysts. Chem. Eng. J. 2007;134:51-7.

[40] Zhang X, Lei H, Wang L, Zhu L, Wei Y, Liu Y, et al. Renewable gasoline-range aromatics and hydrogen-enriched fuel gas from biomass via catalytic microwave-induced pyrolysis. Green Chem. 2015:4029-36.

[41] Luque R, Menéndez JA, Arenillas A, Cot J. Microwave-assisted pyrolysis of biomass feedstocks: the way forward? Energy Environ. Sci. 2012;5:5481-8.

[42] Lidstrom P, Tierney J, Wathey B, Westman J. Microwave assisted organic synthesis - a review. Tetrahedron. 2001;57:9225-83.

[43] Bu Q, Lei H, Wang L, Wei Y, Zhu L, Zhang X, et al. Bio-based phenols and fuel production from catalytic microwave pyrolysis of lignin by activated carbons. Bioresour. Technol. 2014;162:142-7.

[44] Wang L, Lei H, Lee J, Chen S, Tang J, Ahring B. Aromatic hydrocarbons production from packed-bed catalysis coupled with microwave pyrolysis of Douglas fir sawdust pellets. RSC Adv. 2013;3:14609.

[45] Zhang X, Lei H, Yadavalli G, Zhu L, Wei Y, Liu Y. Gasoline-range hydrocarbons produced from microwave-induced pyrolysis of low-density polyethylene over ZSM-5. Fuel. 2015;144:33-42.

[46] Hollander MAd, Wissink M, Makkee M, Moulijn JA. Gasoline conversion reactivity towards cracking with equilibrated FCC and ZSM-5 catalysts. Appl. Catal., A. 2002:85-102.

[47] Xiang Y, Ma L, Lu C, Zhang Q, Li X. Aqueous system for the improved hydrogenation of phenol and its derivatives. Green Chem. 2008;10:939.

[48] Hu H, Qiao M, Wang S, Fan K, Li H, Zong B, et al. Structural and catalytic properties of skeletal Ni catalyst prepared from the rapidly quenched Ni50Al50 alloy. J. Catal. 2004;221:612-8.

[49] Williams CL, Chang C-C, Do P, Nikbin N, Caratzoulas S, Vlachos DG, et al. Cycloaddition of Biomass-Derived Furans for Catalytic Production of Renewablep-Xylene. ACS Catal. 2012;2:935-9.

[50] Brebu M, Ucar S, Vasile C, Yanik J. Co-pyrolysis of pine cone with synthetic polymers. Fuel. 2010;89:1911-8.

[51] Brebu M, Spiridon I. Co-pyrolysis of LignoBoost® lignin with synthetic polymers. Polym. Degrad. Stab. 2012;97:2104-9.

[52] Bai X, Kim KH, Brown RC, Dalluge E, Hutchinson C, Lee YJ, et al. Formation of phenolic oligomers during fast pyrolysis of lignin. Fuel. 2014;128:170-9.

[53] Xue Y, Zhou S, Brown RC, Kelkar A, Bai X. Fast pyrolysis of biomass and waste plastic in a fluidized bed reactor. Fuel. 2015;156:40-6.

[54] Mullen CA, Boateng AA. Catalytic pyrolysis-GC/MS of lignin from several sources. Fuel Process. 37 Technol. 2010;91:1446-58.

38 [55] Carlson TR, Jae J, Huber GW. Mechanistic Insights from Isotopic Studies of Glucose Conversion to 39 Aromatics Over ZSM-5. ChemCatChem. 2009;1:107-10.

40 [56] Zhang H, Carlson TR, Xiao R, Huber GW. Catalytic fast pyrolysis of wood and alcohol mixtures in a 41 fluidized bed reactor. Green Chem. 2012;14:98-110.

42 [57] Op de Beeck B, Dusselier M, Geboers J, Holsbeek J, Morré E, Oswald S, et al. Direct catalytic 
conversion of cellulose to liquid straight-chain alkanes. Energy Environ Sci. 2015;8:230-40.

[58] Keil DE, Warren DA, Jenny MJ, EuDaly JG, Smythe J, Peden-Adams MM. Immunological function in mice exposed to JP-8 jet fuel in utero. Toxicol. Sci. 2003;76:347-56.

[59] Ren S, Lei H, Wang L, Bu Q, Chen S, Wu J, et al. Biofuel production and kinetics analysis for microwave pyrolysis of Douglas fir sawdust pellet. J. Anal. Appl. Pyrolysis. 2012;94:163-9.

[60] Artetxe M, Lopez G, Amutio M, Elordi G, Bilbao J, Olazar M. Light olefins from HDPE cracking in a two-step thermal and catalytic process. Chem. Eng. J. 2012;207-208:27-34.

[61] Artetxe M, Lopez G, Elordi G, Amutio M, Bilbao J, Olazar M. Production of Light Olefins from Polyethylene in a Two-Step Process: Pyrolysis in a Conical Spouted Bed and Downstream HighTemperature Thermal Cracking. Ind. Eng. Chem. Res. 2012;51:13915-23.

[62] Serrano DP, Aguado J, Escola JM, Rodríguez JM, San Miguel G. An investigation into the catalytic cracking of LDPE using Py-GC/MS. J. Anal. Appl. Pyrolysis. 2005;74:370-8.

13 [63] Lopez-Urionabarrenechea A, de Marco I, Caballero BM, Laresgoiti MF, Adrados A. Catalytic stepwise 14 pyrolysis of packaging plastic waste. J. Anal. Appl. Pyrolysis. 2012;96:54-62.

[64] Carlson TR, Cheng Y-T, Jae J, Huber GW. Production of green aromatics and olefins by catalytic fast pyrolysis of wood sawdust. Energy Environ. Sci. 2011:145-61.

[65] Bi P, Wang J, Zhang Y, Jiang P, Wu X, Liu J, et al. From lignin to cycloparaffins and aromatics: Directional synthesis of jet and diesel fuel range biofuels using biomass. Bioresour. Technol. 2015;183:10-7.

[66] Gross BH, Mebane* RC, Armstrong DL. Transfer hydrogenolysis of aromatic alcohols using Raney catalysts and 2-propanol. Appl. Catal., A. 2001;219:281- 9.

[67] Escola JM, Aguado J, Serrano DP, Briones L, Díaz de Tuesta JL, Calvo R, et al. Conversion of Polyethylene into Transportation Fuels by the Combination of Thermal Cracking and Catalytic Hydroreforming over Ni-Supported Hierarchical Beta Zeolite. Energy Fuels. 2012;26:3187-95.

25

26

27 
Table 1 Elemental analyses of Douglas fir sawdust pellets and low-density polyethylene

\begin{tabular}{lcc}
\hline Elemental analysis $(w t \%)$ & Douglas fir sawdust pellets & Low-density polyethylene \\
\hline Carbon & 47.90 & 85.71 \\
Hydrogen & 6.55 & 14.29 \\
Nitrogen & 0.08 & 0 \\
Oxygen $^{\text {a }}$ & 45.57 & 0 \\
${ }^{\text {a Determined by difference }}$ & & \\
\hline
\end{tabular}


Table 2 Experimental design and product yield distribution ${ }^{\mathrm{a}}$

\begin{tabular}{|c|c|c|c|c|c|c|}
\hline \multirow{2}{*}{ Entry $^{b}$} & \multirow{2}{*}{$\begin{array}{l}\text { Catalytic } \\
\text { Temperature }\left({ }^{\circ} \mathrm{C}\right)\end{array}$} & \multirow{2}{*}{$\begin{array}{l}\text { Plastics to } \\
\text { biomass } \\
\text { ratio }\end{array}$} & \multicolumn{4}{|c|}{ Yield (wt $\%)$} \\
\hline & & & Bio-oil & Gas & Char & Coke \\
\hline 1 & 300 & 0.5 & 39.97 & 39.27 & 17.21 & 3.55 \\
\hline 2 & 300 & 1 & 45.32 & 39.93 & 11.86 & 2.89 \\
\hline 3 & 450 & 0.5 & 34.55 & 45.73 & 16.74 & 2.98 \\
\hline 4 & 450 & 1 & 40.24 & 46.07 & 12.02 & 1.67 \\
\hline 5 & 375 & 0.75 & 42.54 & 41.33 & 13.11 & 3.02 \\
\hline 6 & 375 & 0.75 & 41.38 & 42.44 & 13.20 & 2.98 \\
\hline 7 & 375 & 0.75 & 42.09 & 41.93 & 12.87 & 3.11 \\
\hline 8 & 375 & 0.75 & 41.68 & 42.00 & 13.44 & 2.88 \\
\hline 9 & 375 & 0.75 & 41.77 & 42.24 & 13.02 & 2.97 \\
\hline 10 & 375 & 0.4 & 35.22 & 43.61 & 17.57 & 3.60 \\
\hline 11 & 375 & 1.1 & 43.85 & 41.72 & 11.56 & 2.87 \\
\hline 12 & 269 & 0.75 & 42.85 & 40.57 & 13.24 & 3.34 \\
\hline 13 & 481 & 0.75 & 38.23 & 47.56 & 12.78 & 1.43 \\
\hline 14 & 375 & 1.45 & 45.57 & 42.76 & 9.09 & 2.58 \\
\hline 15 & 200 & 0.75 & 47.00 & 36.18 & 13.28 & 3.54 \\
\hline 16 & 500 & 0.75 & 36.47 & 49.56 & 12.95 & 1.02 \\
\hline 17 & 375 & 0 & 30.57 & 43.89 & 21.69 & 3.85 \\
\hline
\end{tabular}

${ }^{a}$ reaction condition: reaction temperature $480{ }^{\circ} \mathrm{C}$; reaction time, 10 mins.

${ }^{\mathrm{b}}$ Entry-1 to Entry-13 were conducted based on central composite design; Entry-14 and Entry-16 were added as the controls; Entry-17 is the control in the absence of plastics. 
1

2

3

4

5

Table 3 Textural properties of well-promoted ZSM-5 and home-made Raney Ni catalysts ${ }^{\mathrm{a}}$

\begin{tabular}{lccccc} 
& $S_{\text {BET }}$ & $V_{\text {pore }}$ & $S_{\text {pore }}$ & $d_{\text {pore }}$ & $T_{\text {acidity }}$ \\
\cline { 2 - 6 } & $\left(\mathrm{m}^{2} / \mathrm{g}\right)$ & $\left(\mathrm{cm}^{3} / \mathrm{g}\right)$ & $\left(\mathrm{m}^{2} / \mathrm{g}\right)$ & $\mathrm{nm}$ & $\mathrm{mmol} \mathrm{NH}_{3} / \mathrm{g}$ \\
\hline Parent ZSM-5 & 386.9 & 0.078 & 55.3 & 5.7 & 0.20 \\
Well-promoted ZSM-5 & 396.2 & 0.097 & 74.1 & 5.2 & 0.18 \\
Ni-Al alloy & 0.35 & 0 & 0 & 0 & - \\
Home-made Raney Ni & 52.4 & 0.034 & 35.8 & 3.8 & -
\end{tabular}

${ }^{a} S_{\mathrm{BET}}$ : BET surface area; $V_{\text {pore }}$ : pore volume; $S_{\text {pore }}$ : pore surface area; $d_{\text {pore }}$ : average pore size; $T_{\text {acidity: }}$ total acidity.
6

7

8

9

10

11

12

13

14

15

16

17

18

19

20

21

22 
Table 4 Detailed carbon yield distribution and product carbon selectivity as a function of catalytic temperature $^{\mathrm{a}}$

\begin{tabular}{|c|c|c|c|c|c|}
\hline & \multicolumn{5}{|c|}{ Catalytic temperature $\left({ }^{\circ} \mathrm{C}\right)$} \\
\hline & 200 & 269 & 375 & 481 & 500 \\
\hline \multicolumn{6}{|c|}{ Overall carbon yield (C mol\%) } \\
\hline Liquid organics & 42.23 & 40.84 & 40.54 & 36.22 & 34.65 \\
\hline Char & 18.02 & 17.56 & 17.87 & 18.34 & 17.67 \\
\hline Coke & 5.98 & 5.42 & 4.23 & 2.78 & 1.46 \\
\hline $\mathrm{Gas}^{\mathrm{b}}$ & 33.77 & 36.18 & 37.36 & 42.66 & 46.22 \\
\hline \multicolumn{6}{|c|}{ Liquid carbon selectivity (C mol\%) } \\
\hline Toluene & 4.97 & 6.48 & 10.72 & 14.62 & 18.42 \\
\hline Ethylbenzene & 1.18 & 1.63 & 2.29 & 2.66 & 1.99 \\
\hline$p$-xylene $/ m$-xylene & 2.50 & 7.67 & 18.78 & 25.27 & 27.41 \\
\hline Trimethylbenzene & 4.35 & 16.74 & 17.13 & 10.41 & 9.01 \\
\hline Indane & 0.39 & 0.72 & - & 2.24 & 2.00 \\
\hline Indene & 1.60 & 1.30 & 0.79 & 1.91 & 1.59 \\
\hline Phenol & 1.92 & 1.78 & 1.51 & 1.46 & 1.19 \\
\hline$p$-cresol $/ m$-cresol & 2.96 & 2.19 & 1.74 & 1.52 & 1.09 \\
\hline Naphthalene & 5.51 & 3.90 & 5.33 & 6.96 & 6.43 \\
\hline 1-methylnaphthalene & - & 1.12 & 5.29 & 7.08 & 7.50 \\
\hline 2-methylnaphthalene & - & 0.97 & 2.13 & 0.90 & 0.95 \\
\hline Anthracene & - & - & 0.85 & 1.29 & 1.28 \\
\hline Pyrene & - & 0.12 & - & 0.09 & 0.24 \\
\hline \multicolumn{6}{|c|}{ Gaseous carbon selectivity (C mol\%) } \\
\hline Methane & 12.45 & 13.11 & 13.65 & 14.02 & 14.25 \\
\hline Carbon monoxide & 30.32 & 26.54 & 16.43 & 18.61 & 20.11 \\
\hline Carbon dioxide & 22.45 & 20.45 & 18.98 & 20.10 & 20.32 \\
\hline Ethylene & 18.45 & 24.20 & 31.45 & 36.54 & 40.22 \\
\hline Ethane & 7.18 & 8.43 & 11.23 & 5.09 & 2.91 \\
\hline Propane & 9.15 & 7.27 & 8.26 & 5.64 & 2.19 \\
\hline \multicolumn{6}{|c|}{$\begin{array}{l}\text { Reaction condition: Catalyst, } 20 \mathrm{wt} \% \text { with respect to feed; Plastics, } 75 \mathrm{wt} \% \text { with respect to } \\
\text { biomass; Reaction temperature, } 480{ }^{\circ} \mathrm{C} \text {; Reaction time, } 10 \mathrm{~min} \text {. } \\
\text { b Determined by difference }\end{array}$} \\
\hline
\end{tabular}

4 
Table 5 Detailed carbon yield distribution and product carbon selectivity on the basis of plastics to biomass ratio $^{\text {a }}$

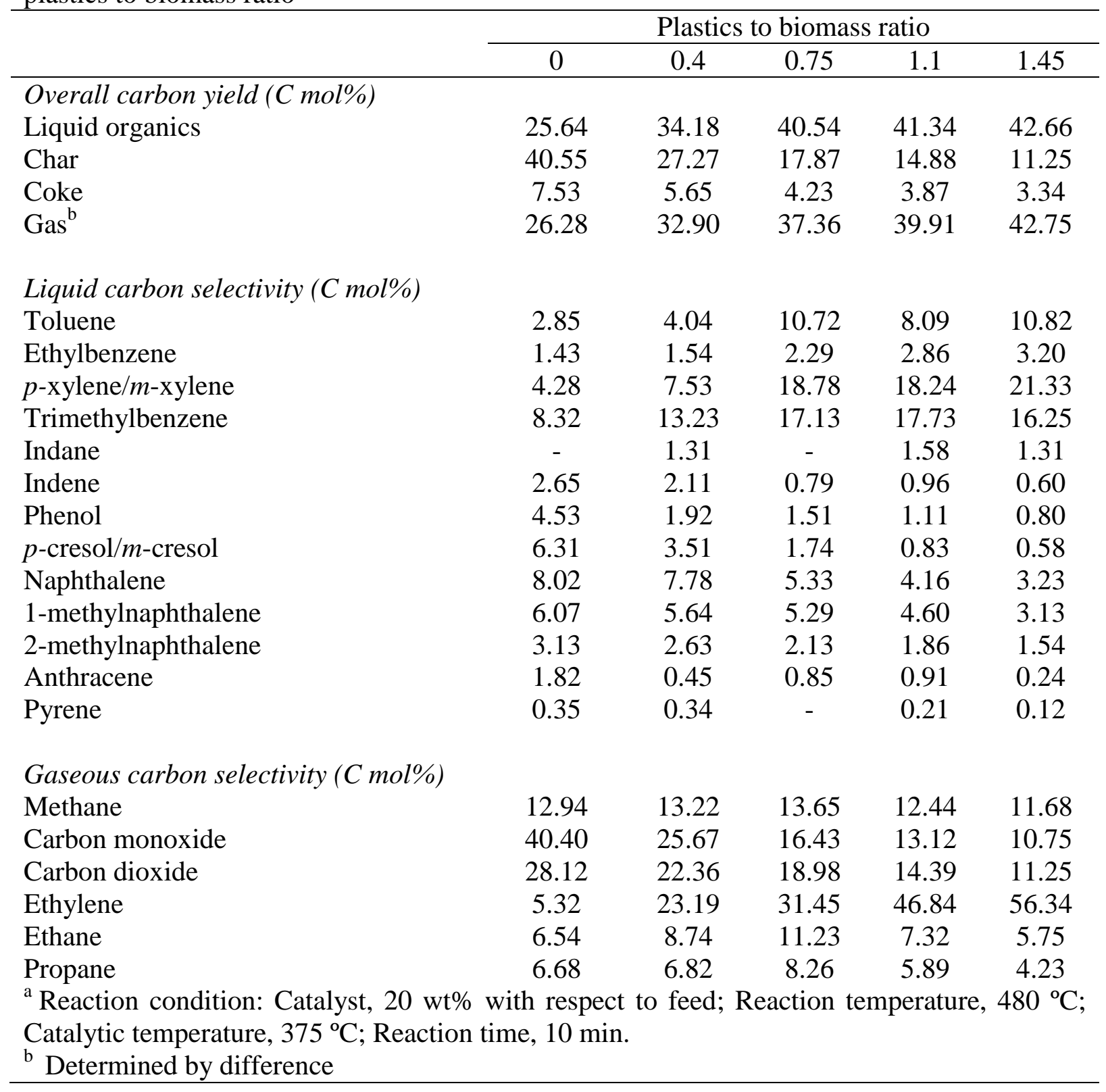

4 
Table 6 Detailed carbon yield distribution and product carbon selectivity with respect to catalyst reused times $^{\mathrm{a}}$

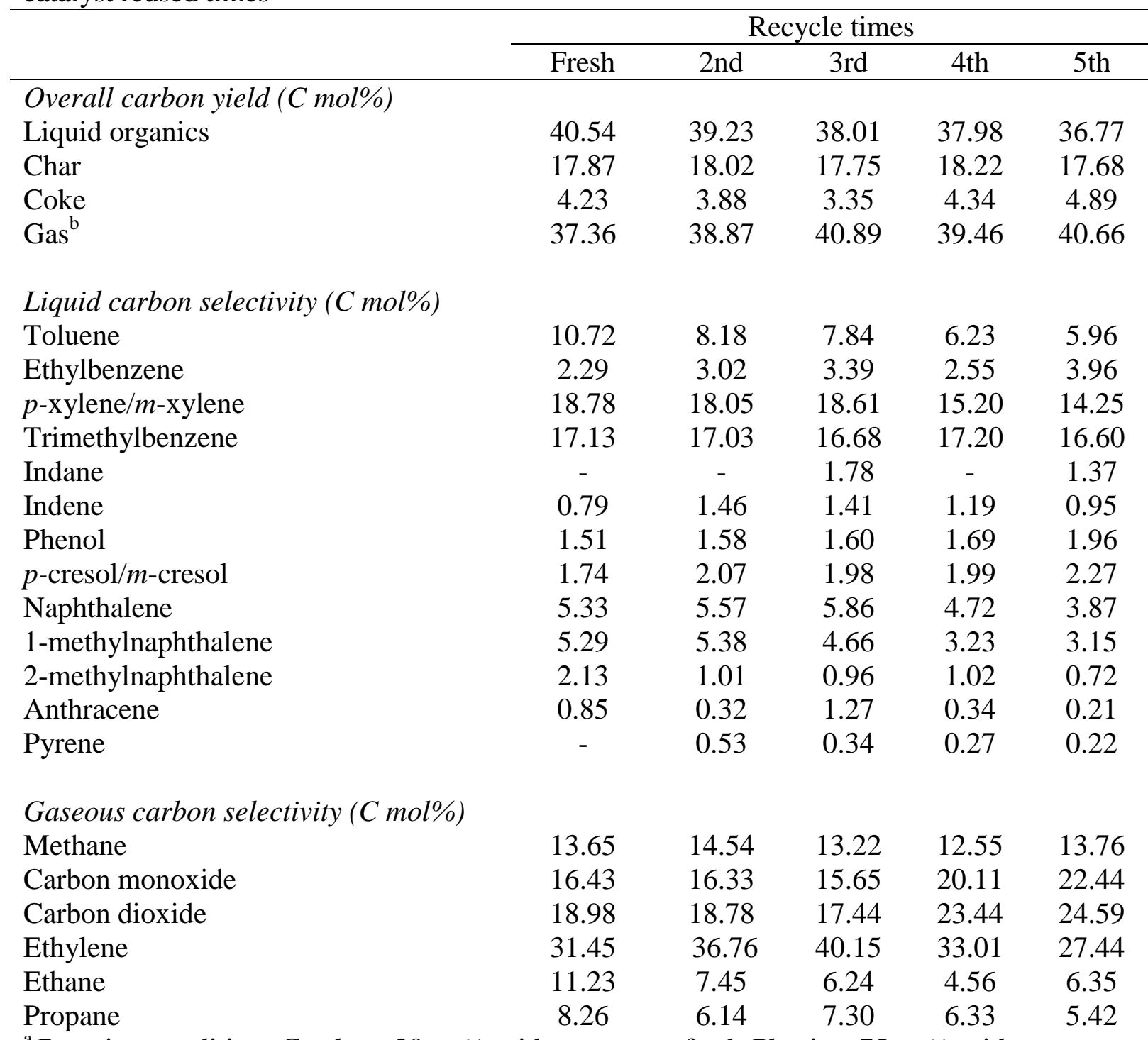

${ }^{a}$ Reaction condition: Catalyst, $20 \mathrm{wt} \%$ with respect to feed; Plastics, 75 wt $\%$ with respect to biomass; Reaction temperature, $480{ }^{\circ} \mathrm{C}$; Catalytic temperature, $375{ }^{\circ} \mathrm{C}$; Reaction time, 10 min.

b Determined by difference 
Table 7 Products distribution and partial alkanes' carbon selectivity for hydrogenation of diverse liquid organics ${ }^{\mathrm{a}}$

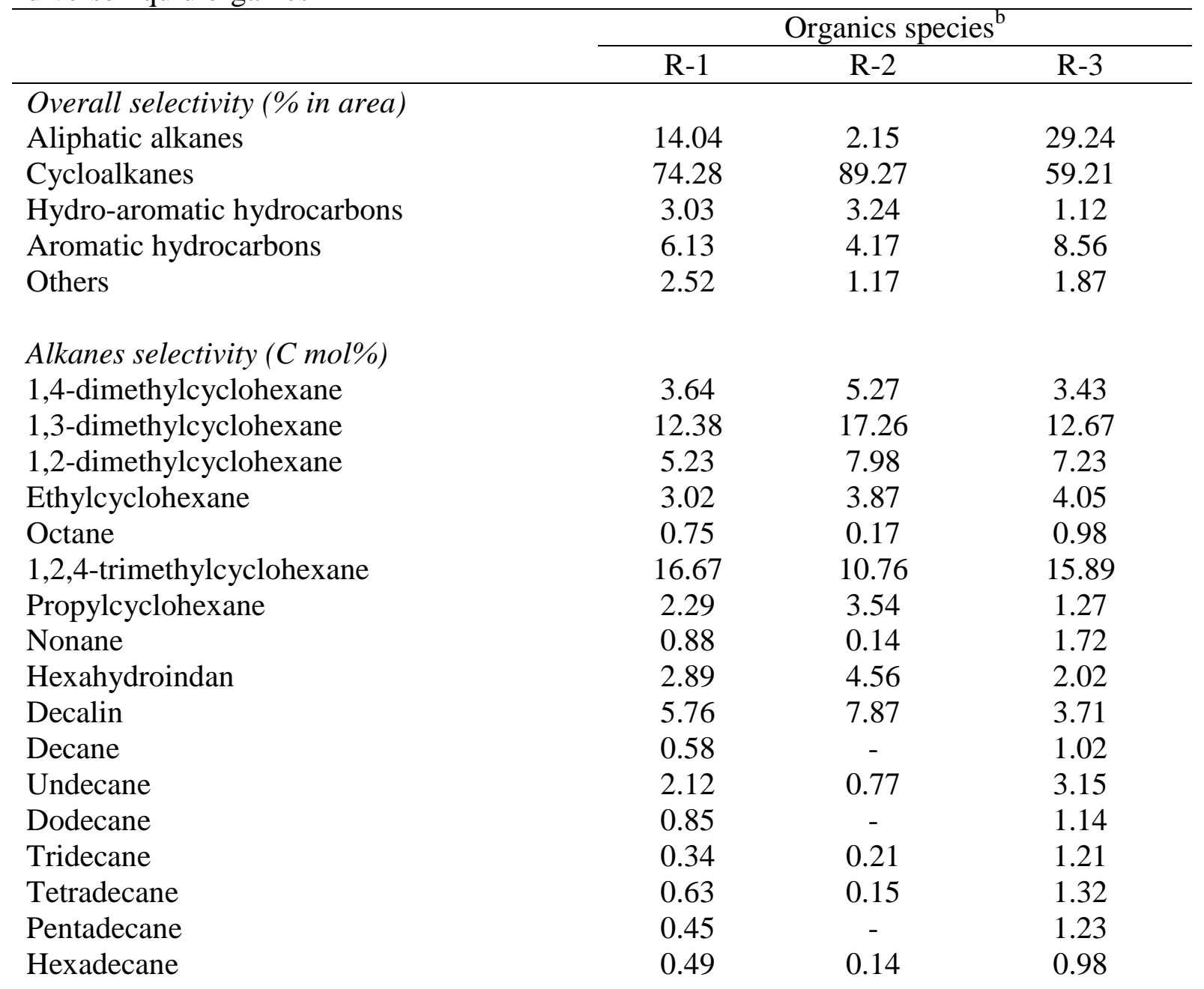

${ }^{a}$ Reaction condition: Initial pressure, 500 psi; Raney Ni catalyst, 20 wt\% with respect to reactant mass; Reaction temperature, $200^{\circ} \mathrm{C}$; Reaction time, $2 \mathrm{~h}$.

${ }^{\mathrm{b}} \mathrm{R}-1$ : reactant from the experiment conducted at catalytic temperature of $375{ }^{\circ} \mathrm{C}$ with plastics to biomass ratio of 0.75 ; R-2: reactant from the experiment conducted at catalytic temperature of $500{ }^{\circ} \mathrm{C}$ with plastics to biomass ratio of 0.75 ; R-3: reactant from the experiment conducted at catalytic temperature of 375 with plastics to biomass ratio of 1.45 . 
Fig. $1 \mathrm{NH}_{3}$-TPD profiles of parent ZSM-5 and well-promoted ZSM-5 catalyst.

Fig. 2 The XRD patterns of the Ni-Al alloy powder and home-made Raney Ni catalyst.

Fig. 3 SEM images of Ni-Al alloy powder (A) and home-made Raney Ni catalyst (B).

10

Fig. 4 The overall carbon yields regarding product distribution from co-feed catalytic microwave pyrolysis in light of catalytic temperature at the same plastics to biomass ratio (0.75).

Fig. 5 The overall carbon yields regarding product distribution from co-feed catalytic microwave pyrolysis as a function of plastics to biomass ratio at the same catalytic temperature $\left(375^{\circ} \mathrm{C}\right)$.

16

Fig. 6 The overall carbon yields regarding product distribution from co-feed catalytic microwave pyrolysis on the basis of catalyst recycle times at the catalytic temperature of $375{ }^{\circ} \mathrm{C}$ with the plastics to biomass ratio of 0.75 .

Fig. 7 Proposed reaction pathways for the conversion of lignocellulosic biomass and plastics into 22 jet fuel range alkanes. 


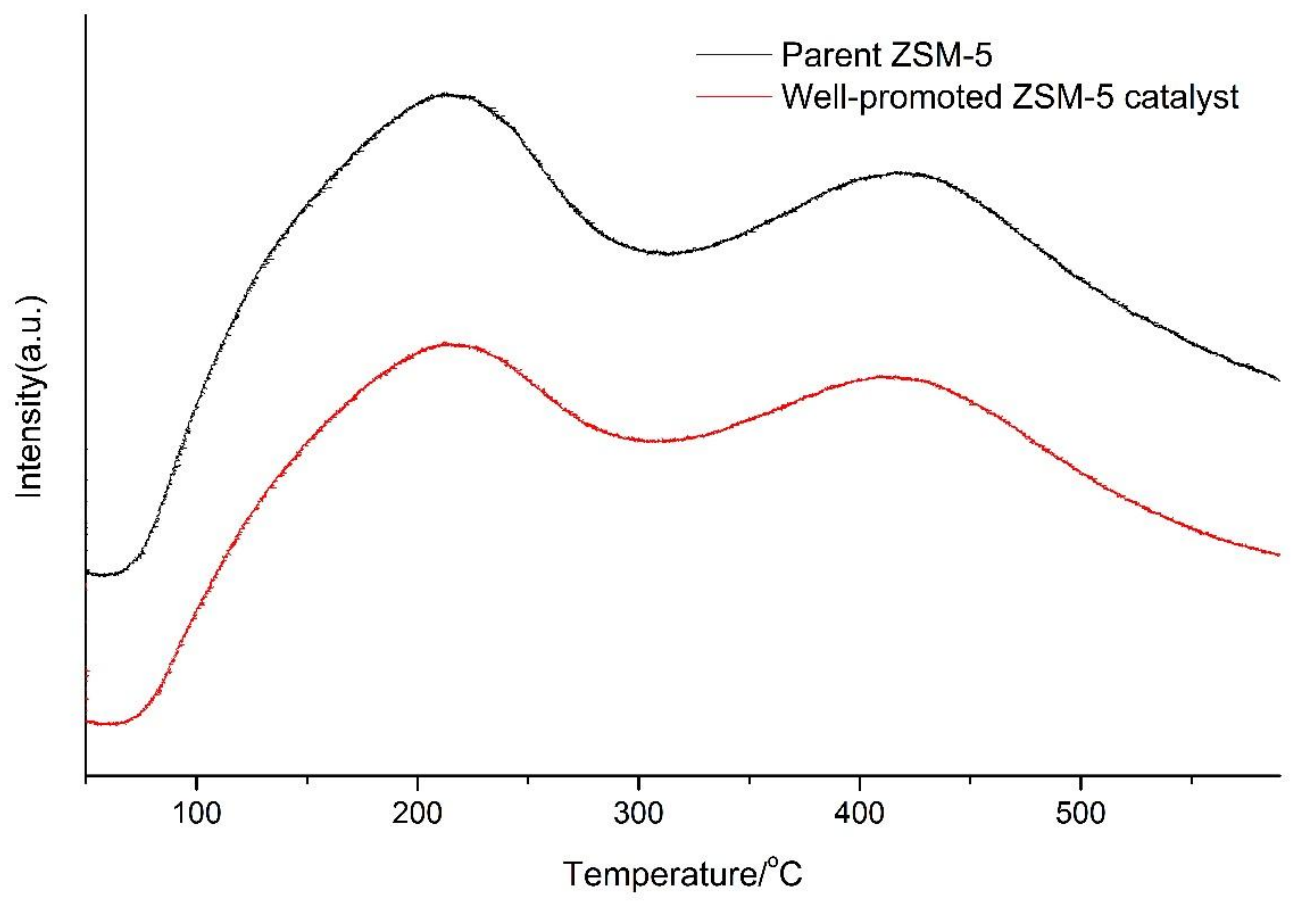

11 Fig. $1 \mathrm{NH}_{3}$-TPD profiles of parent ZSM-5 and well-promoted ZSM-5 catalyst.

12

13

14

15

16

17 


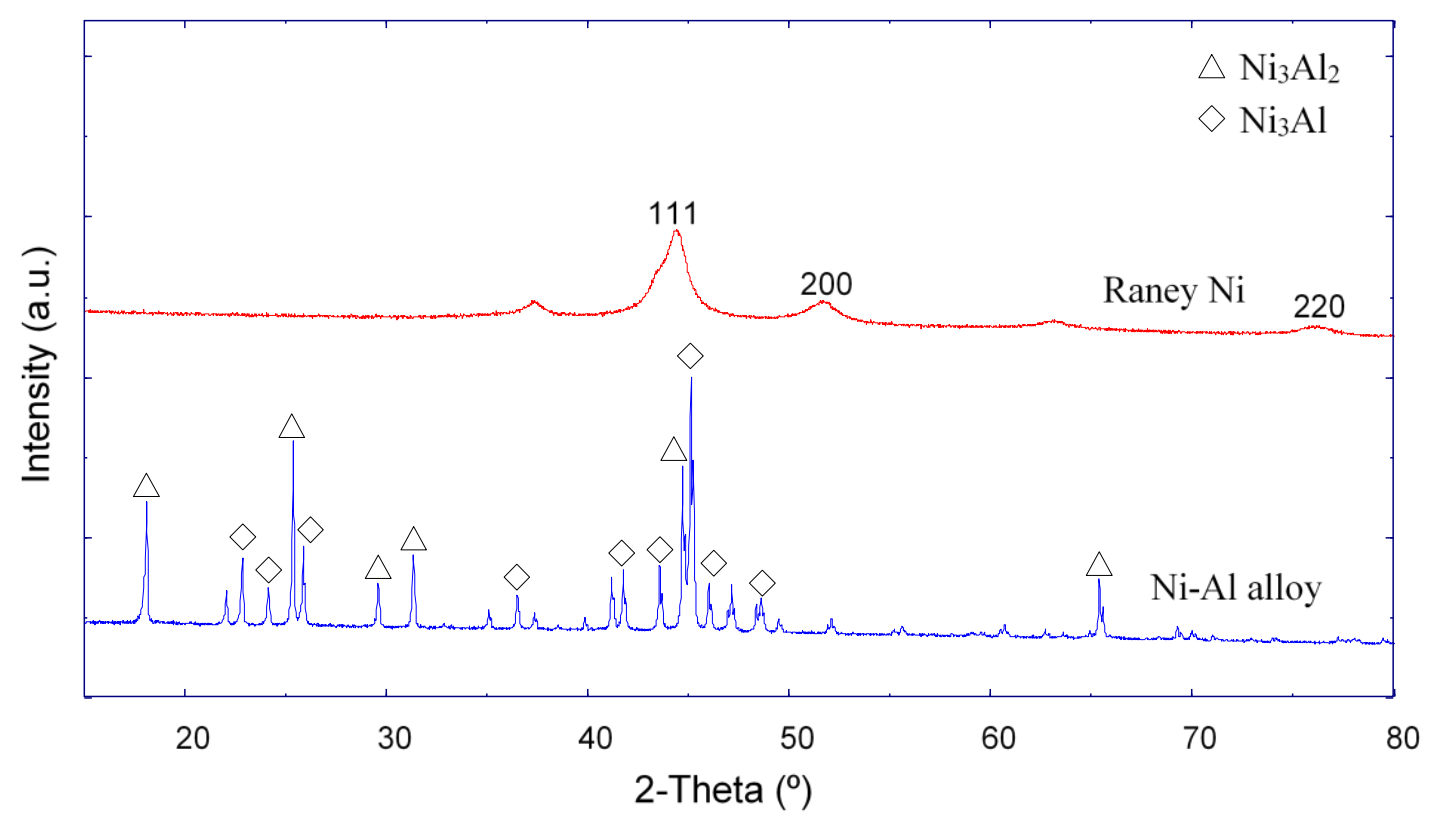

9

10

11

Fig. 2 The XRD patterns of the Ni-Al alloy powder and home-made Raney Ni catalyst.

12

13

14

15

16

17

18

19

20 

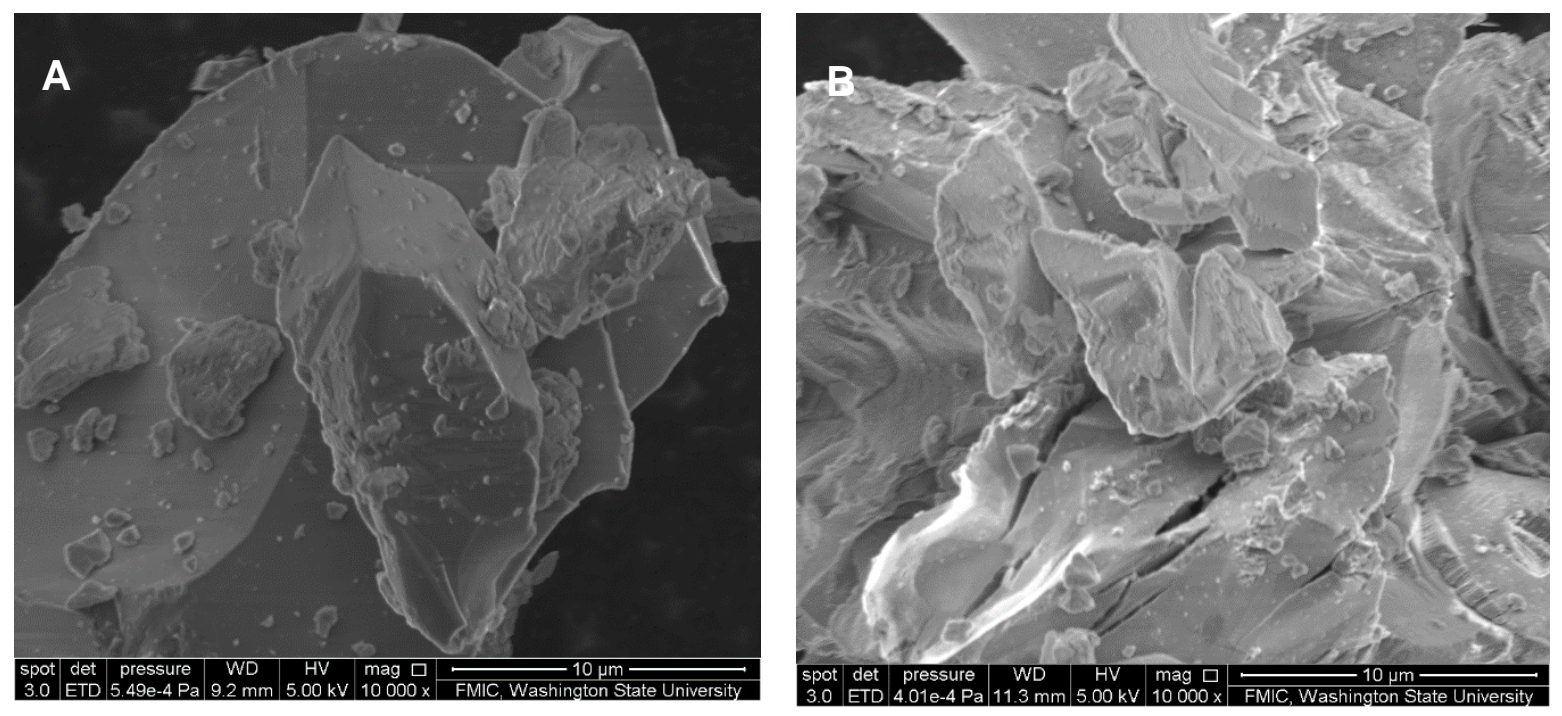

13 Fig. 3 SEM images of Ni-Al alloy powder (A) and home-made Raney Ni catalyst (B).

14

15

16

17

18

19

20

21 


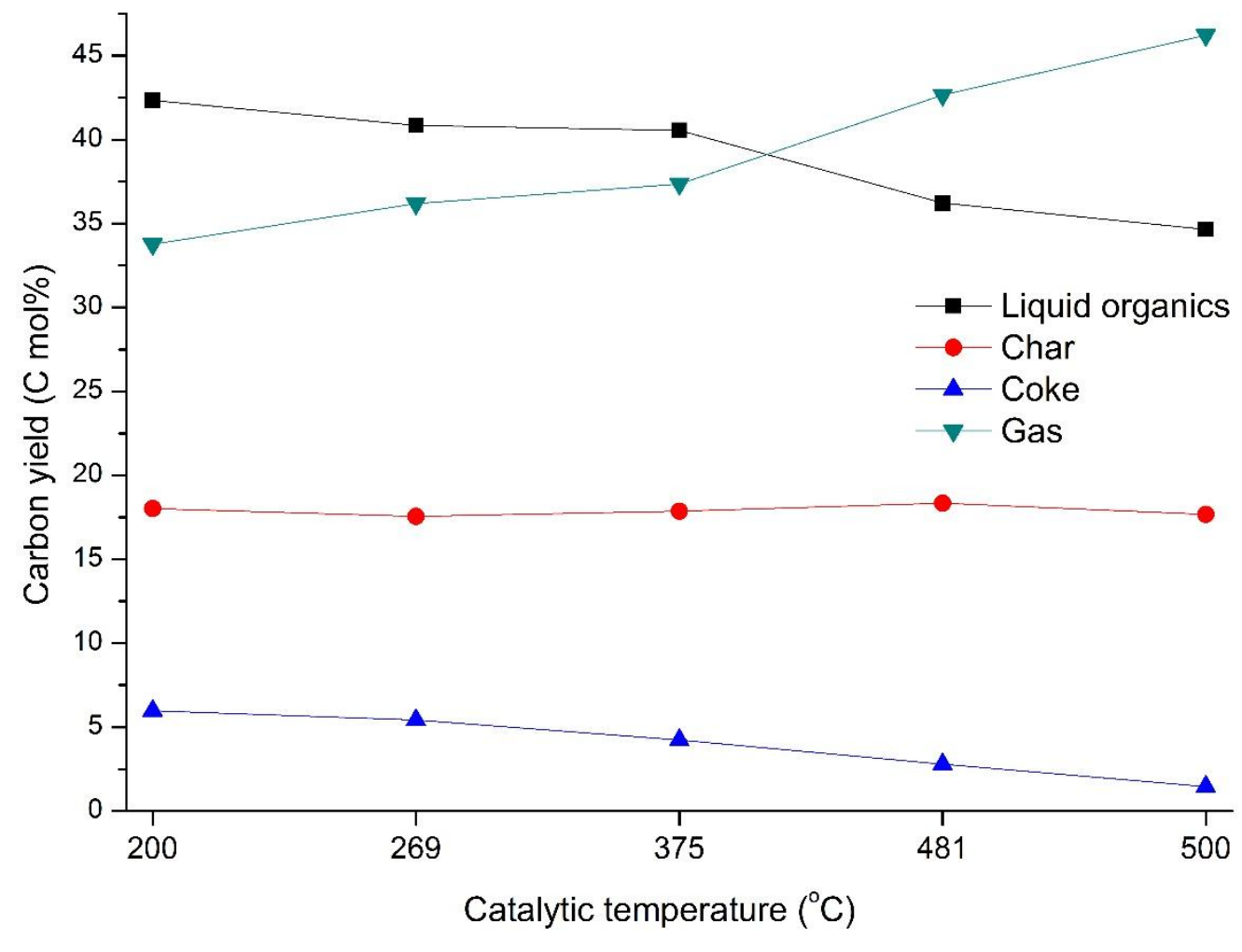

9

Fig. 4 The overall carbon yields regarding product distribution from co-feed catalytic microwave pyrolysis in light of catalytic temperature at the same plastics to biomass ratio (0.75).

12

13

14

15

16

17

18 


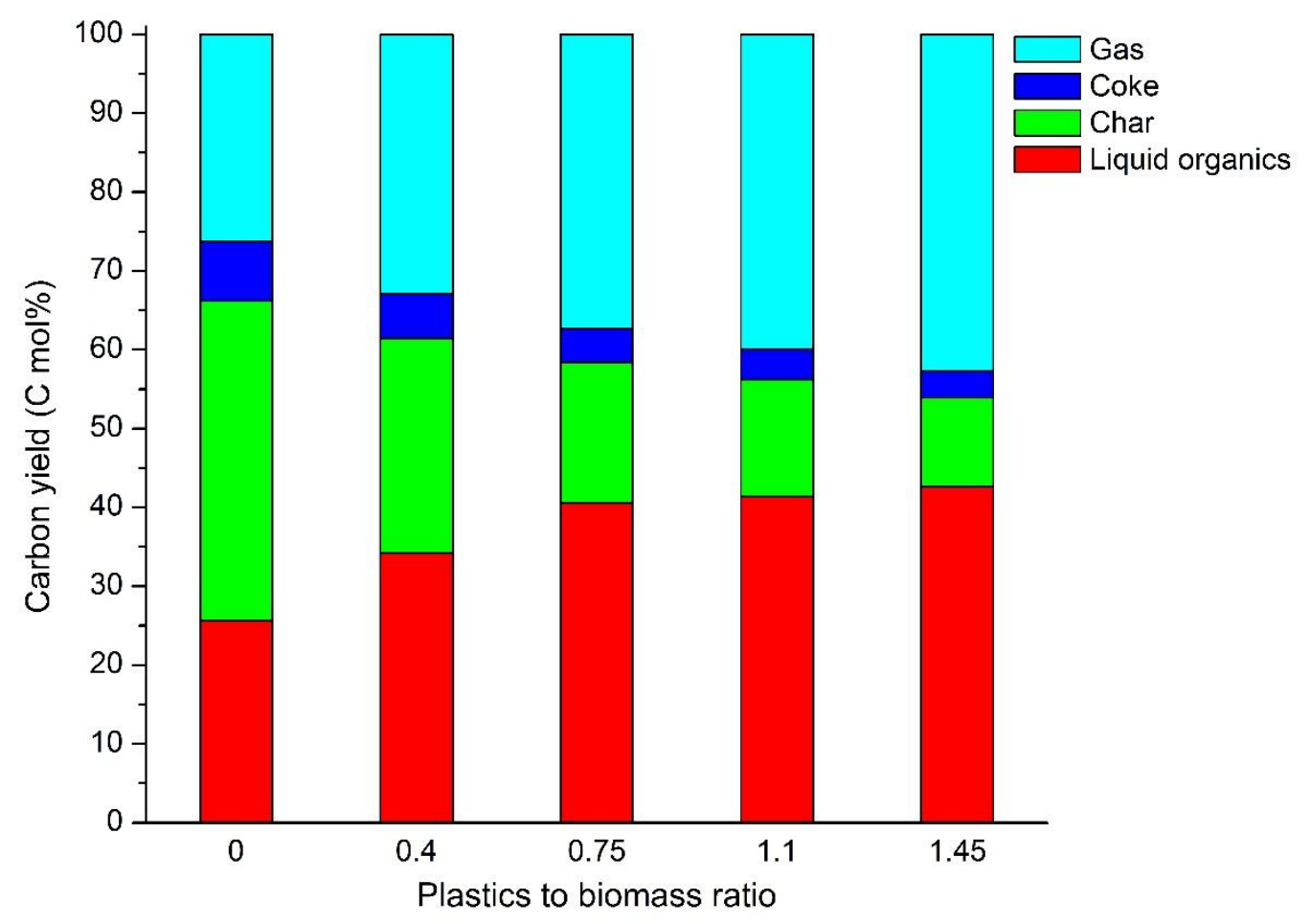

Fig. 5 The overall carbon yields regarding product distribution from co-feed catalytic microwave 


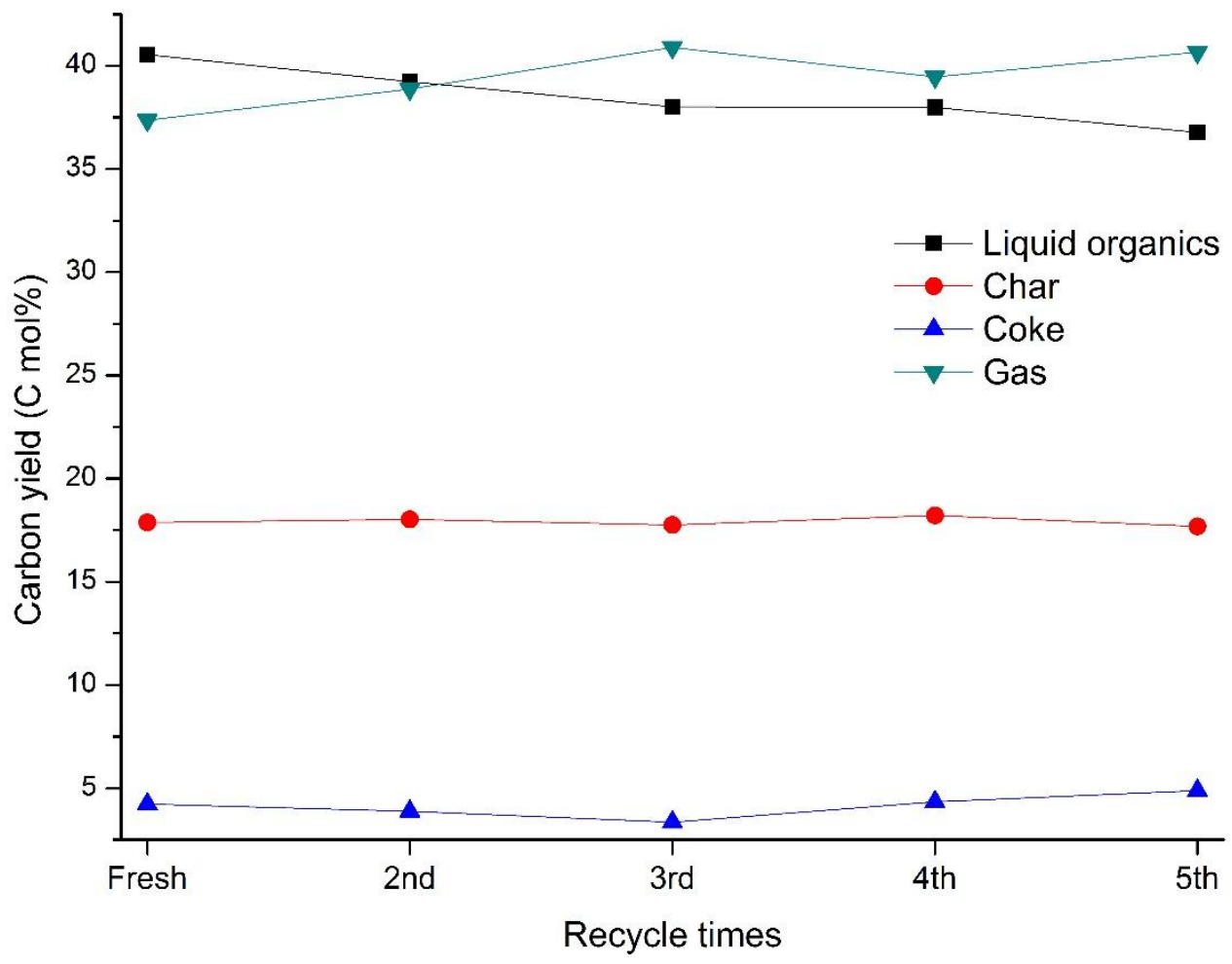

10

11

12

13

14 


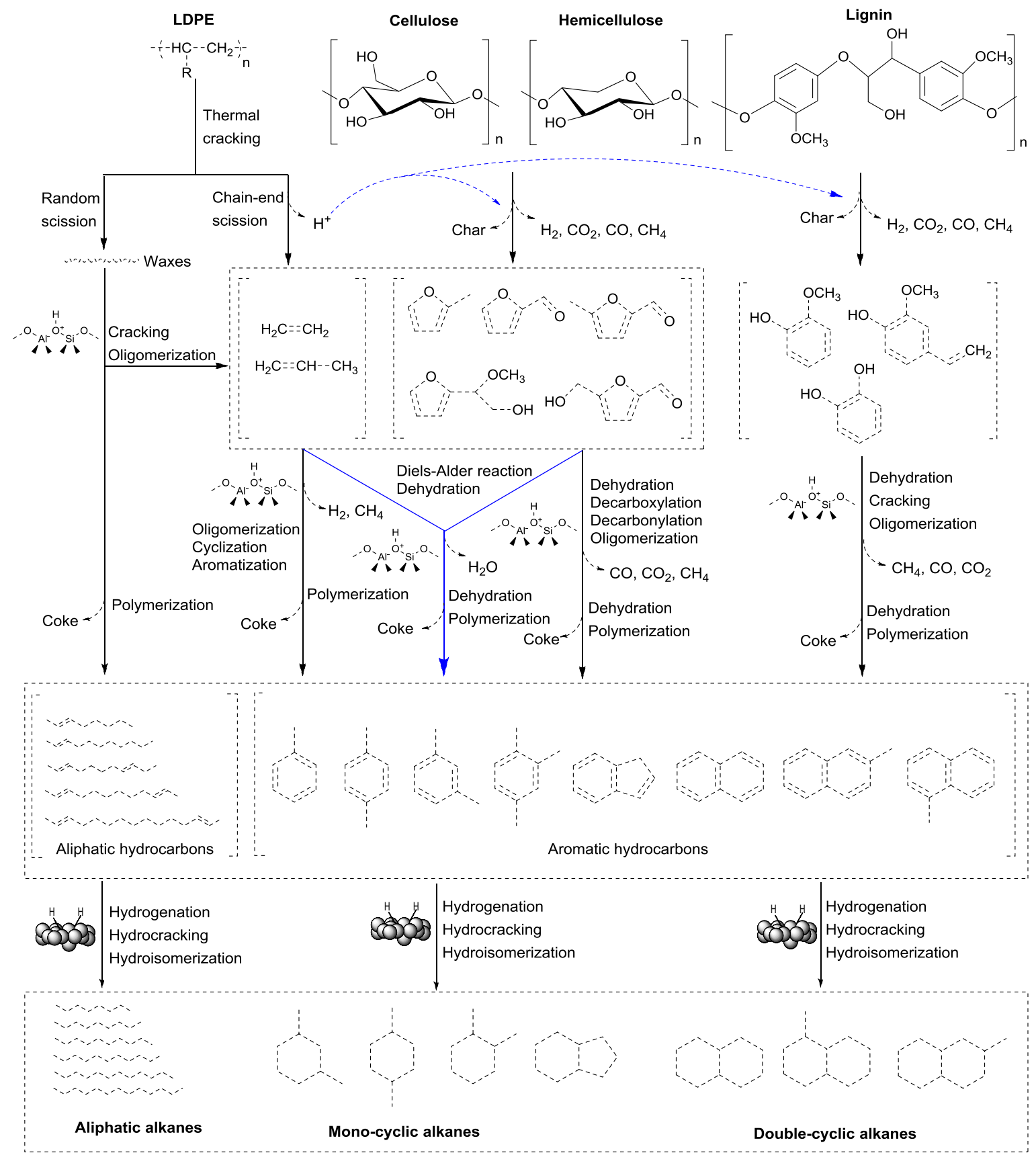

2 Fig. 7 Proposed reaction pathways for the conversion of lignocellulosic biomass and plastics into 3 jet fuel range alkanes. 
Graphical Abstract

Improvement of renewable alkanes for jet fuels from co-feed catalytic microwaveassisted pyrolysis and hydrogenation process

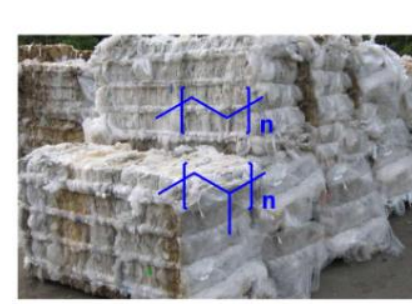

Waste plastics
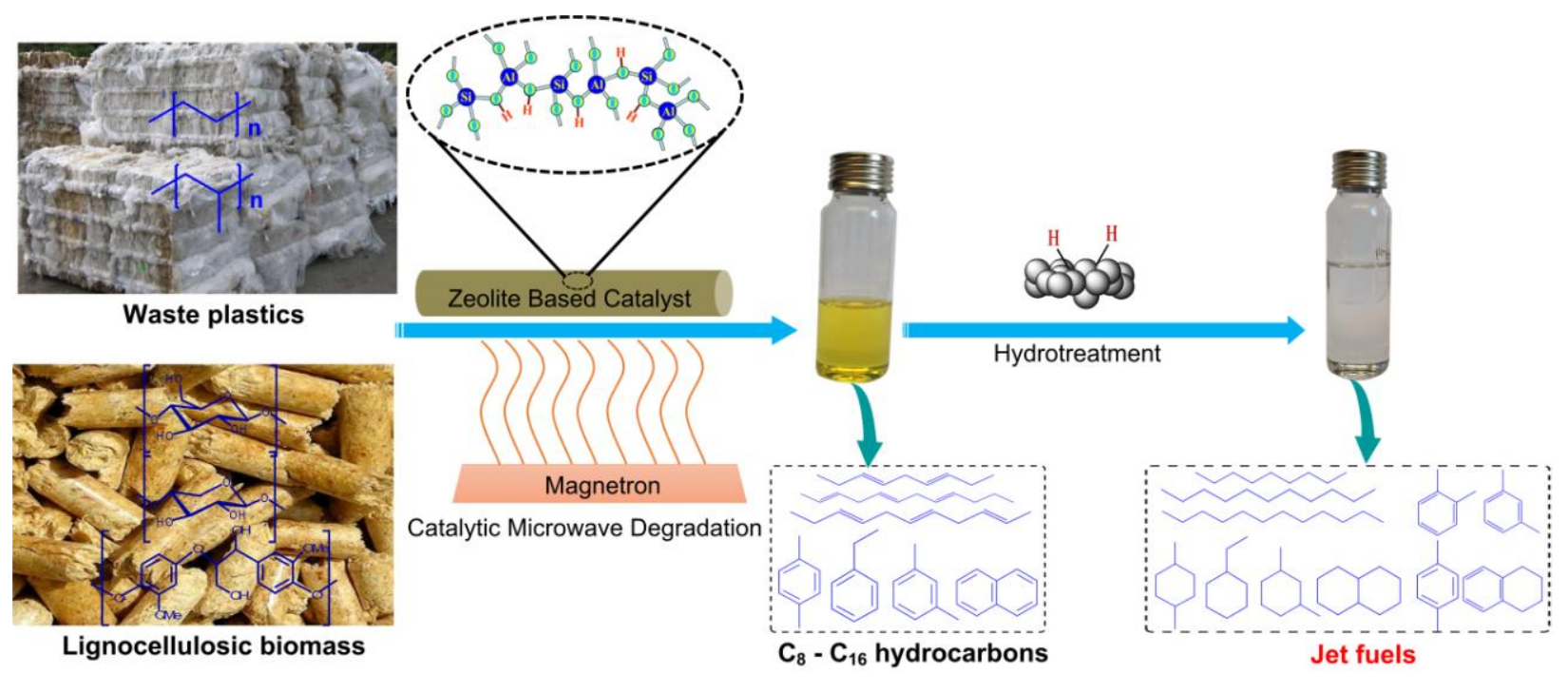

Lignocellulosic biomass

Catalytic Microwave Degradation

$$
\mathrm{C}_{8}-\mathrm{C}_{16} \text { hydrocarbons }
$$

Jet fuels

\section{.}
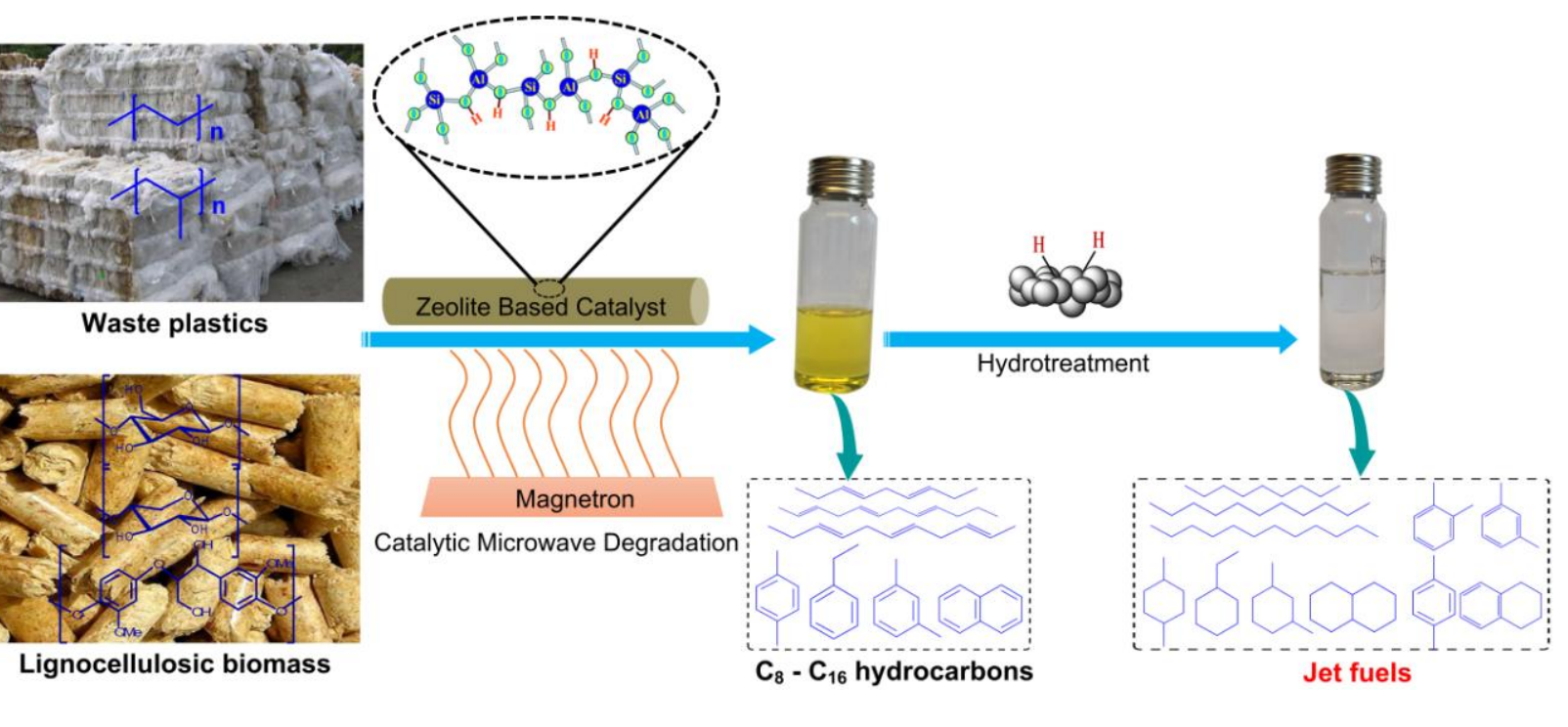